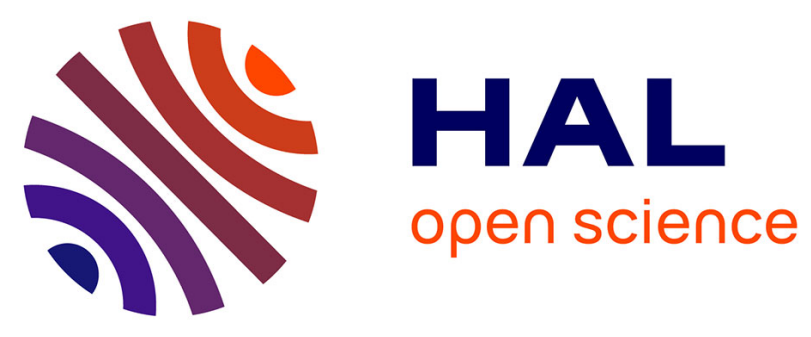

\title{
Stabilization of Ni2+ dimers in hexacyano Mo6 cluster-based Prussian blue derivatives: Experimental and theoretical investigations of magnetic properties
}

G. Daigre, K. Costuas, M.S. Tarasenko, A.Y. Ledneva, N.G. Naumov, P. Lemoine, T. Guizouarn, Y. Molard, M. Amela-Cortes, Nathalie Audebrand, et al.

\section{To cite this version:}

G. Daigre, K. Costuas, M.S. Tarasenko, A.Y. Ledneva, N.G. Naumov, et al.. Stabilization of Ni2+ dimers in hexacyano Mo6 cluster-based Prussian blue derivatives: Experimental and theoretical investigations of magnetic properties. Dalton Transactions, 2018, 47 (4), pp.1122-1130. $10.1039 / \mathrm{c} 7 \mathrm{dt} 02748 \mathrm{f}$. hal-01713505

HAL Id: hal-01713505

https://hal-univ-rennes1.archives-ouvertes.fr/hal-01713505

Submitted on 16 May 2018

HAL is a multi-disciplinary open access archive for the deposit and dissemination of scientific research documents, whether they are published or not. The documents may come from teaching and research institutions in France or abroad, or from public or private research centers.
L'archive ouverte pluridisciplinaire HAL, est destinée au dépôt et à la diffusion de documents scientifiques de niveau recherche, publiés ou non, émanant des établissements d'enseignement et de recherche français ou étrangers, des laboratoires publics ou privés. 


\title{
Hexacyano molybdenum clusters chalcohalides and divalent cations $\left(\mathrm{Zn}^{2+}\right.$ and $\left.\mathrm{Cu}^{2+}\right)$ for the design of extended polymeric and supramolecular frameworks
}

\author{
Gilles Daigre $^{\mathrm{a}}$, Maria S. Tarasenko ${ }^{\mathrm{b}}$, Alexandra Yu. Ledneva ${ }^{\mathrm{b}}$, Nikolai G. Naumov ${ }^{\mathrm{b}, \mathrm{c}}$, Nathalie \\ Audebrand $*^{\mathrm{a}}$, Stéphane Cordier*a
}

${ }^{a}$ Institut des Sciences Chimiques de Rennes UMR 6226 CNRS-Université de Rennes1, France; ${ }^{b}$ Nikolaev Institute of Inorganic Chemistry, 3 Acad.Lavrentiev pr., 630090 Novosibirsk, Russia ; ' Novosibirsk State University, Pirogova str.2, 630090 Novosibirsk, Russia.

Abstract: Two new octahedral molybdenum cyanide cluster compounds, namely $\left[\left\{\mathrm{Zn}\left(\mathrm{H}_{2} \mathrm{O}\right)(\mathrm{en})_{2}\right\}\left\{\mathrm{Zn}\left(\mathrm{en}_{2}\right\}-\mathrm{Mo}_{6} \mathrm{~B}_{\mathrm{r6}} \mathrm{Se} \mathrm{C}_{2}(\mathrm{CN})_{6}\right] \cdot 2 \mathrm{H}_{2} \mathrm{O}(\mathbf{1})\right.$ and $\left[\left\{\mathrm{Cu}\left(\mathrm{H}_{2} \mathrm{O}\right)(\mathrm{en})_{2}\right\}_{2} \mathrm{Mo}_{6} \mathrm{Br}_{6} \mathrm{Se}_{2}(\mathrm{CN})_{6}\right] \cdot 2 \mathrm{H}_{2} \mathrm{O}$ (2) (en = ethylenediamine), have been synthetized as single crystals. 1 was obtained by slow diffusion of a solution containing $\left[\mathrm{Mo}_{6} \mathrm{Br}_{6} \mathrm{Se}_{2}(\mathrm{CN})_{6}\right]^{4-}$ cluster units into a solution of zinc acetate and ethylenediamine. 2 was obtained by slow diffusion of an ammonia solution of $\left[\mathrm{Mo}_{6} \mathrm{Br}_{6} \mathrm{Se}_{2}(\mathrm{CN})_{6}\right]^{4-}$ cluster units into a solution of cupper chloride and ethylenediamine. Both compounds were structurally characterized by single-crystal X-ray diffraction analysis. $\mathbf{1}$ is a bimetallic one dimensional coordination compound and it crystallizes in the orthorhombic system $\left(P 2_{1} 2_{1} 2_{1} ; Z=4 ; a=10.675(1) \AA, b=15.073(2) \AA, c=25.812(4) \AA\right.$, $V=4153.3(1)$ $\AA^{3}$ at $\left.\mathrm{T}=150 \mathrm{~K}\right) .2$ is a bimetallic $\mathrm{H}$ bonded three dimensional compound and it crystallizes in the monoclinic system $\left(P 2_{1} / c ; \mathrm{Z}=2\right.$; $a=9.4281(4) \AA, b=11.0220(5) \AA, c=21.1494(9) \AA, \beta=100.053(2)^{\circ}, V=2164.03(16) \AA^{3}$ at $\left.\mathrm{T}=296 \mathrm{~K}\right)$.

Keywords: Clusters compounds / Cyanides / Ethylenediamine / Divalent cation / Molybdenum / Single-crystal Structure / Extended Framework / Supramolecular Framework

\section{INTRODUCTION}

Prussian Blue is well known as a strong blue pigment for more than a century but its crystallographic structure was solved from single-crystal X-ray diffraction data only in 1977 by Ludi et al. [1]. The authors showed that the compound is based on trivalent and divalent $\mathrm{Fe}$ cations leading to $\mathrm{Fe}^{\mathrm{III}}{ }_{4}\left[\mathrm{Fe}^{\mathrm{II}}(\mathrm{CN})_{6}\right]_{3} \cdot \mathrm{xH}_{2} \mathrm{O}$ with $\mathrm{x}=14-16$. It crystallizes in the cubic system and is characterized by missing $\left[\mathrm{Fe}^{\mathrm{II}}(\mathrm{CN})_{6}\right]$ groups leading to a disordered structure. Following this discovery many works have been made in order to obtain Prussian Blue analogues. We can cite, among others, groups of Zhao in China or Verdaguer in France for their works, on one hand, on electroactive thin film of $\mathrm{Pd}^{+\mathrm{HI}}$ based Prussian Blue analogues [2] and, on the other hand, on magnetic properties of $\mathrm{Cr}^{+I I I}$-based compounds [3]. Thereafter, at the same time, the Fedorov group in Russia and the Long group in the United States had the brilliant idea to replace the $\left[\mathrm{M}(\mathrm{CN})_{6}\right]^{3-/ 4-}$ complex by a $\left[\operatorname{Re}_{6} \mathrm{~S}_{8}{ }_{8}(\mathrm{CN})_{6}^{\mathrm{a}}\right]^{3-14-}$ cluster unit thinking that it would be possible to obtain new compounds with similar architecture than that of Prussian Blue due to similar orthogonal disposition of $\mathrm{CN}$ groups in iron complexes and in $\mathrm{Re}_{6}$ cyanides. Enhanced porosity should result from the greater size of the hexacyano-clusters comparing to their mononuclear-based hexacyanides analogues [4-6]. It has been highlighted that the nature of the counter cations and their solvated species along with the charge of $\operatorname{Re}_{6}$ cluster unit (3- or 4-) are the driving forces for the formation of structures with defined dimensionalities. Indeed a wide library of compounds with fascinating structures is reported in the literature [4-11]. Then, the Fedorov group, as well as the Kim group in Korea, continued the research on this topic and they obtained numerous compounds based on $\mathrm{Re}_{6}$ metal atom cluster cyanides and transition metal complexed by chelating ligands [12-16]. Indeed, it is well known that reactions between $\left[\operatorname{Re}_{6} \mathrm{Q}_{8}^{\mathrm{i}}(\mathrm{CN})_{6}^{\mathrm{a}}\right]^{3-/ 4-}(\mathrm{Q}=\mathrm{S}$, Se $)$ and transition metals often lead to close-packed structure [9, 17] or interpenetrating frameworks $[5,18]$. A way to prevent compact structures and meanwhile to favor porosity is to include large counter cations as ammoniu ${ }^{1} \mathrm{~m}$ salt [19-21] or to use chelating ligands as ethylenediamine [22-23] which also permits to control the 0-, 1-, 2- or 3-D dimensionality of the resulting compounds. It is worth noting that when obtained compounds are molecular complexes, their structural

\footnotetext{
* Prof. N. Audebrand, 263 Av. Général Leclerc, CS 74205, 35042 Rennes Cedex, France +33223235714, E-Mail: nathalie.audebrand@univrennes1.fr; *Dr. S. Cordier, 263 Av. Général Leclerc, CS 74205, 35042 Rennes Cedex, France, +33223236536, E-Mail: stephane.cordier@univrennes1.fr
} 
cohesion is mainly ensured by hydrogen bonds [19, 24]. If extended polymeric compounds have been obtained in the chemistry of $\operatorname{Re}_{6}$ octahedral clusters and more recently in the chemistry of $\mathrm{Nb}_{6}$ octahedral clusters [25-27], very few is known about $\mathrm{Mo}_{6}$ hexacyano homologous. Hitherto only one layered compound based on $\left[\mathrm{Mo}_{6} \mathrm{Br}_{8}^{\mathrm{i}}(\mathrm{CN})_{6}^{\mathrm{a}}\right]^{2-}$, namely [trans- $\left.\mathrm{Cd}\left(\mathrm{H}_{2} \mathrm{O}\right)_{2}\right]\left[\mathrm{Mo}_{6} \mathrm{Br}_{8}(\mathrm{CN})_{6}\right]$, has been reported so far [28]. On the other hand, no extended polymeric frameworks based on $\left[\mathrm{Mo}_{6} \mathrm{Br}_{6}^{\mathrm{i}} \mathrm{Q}_{2}^{\mathrm{i}}(\mathrm{CN})_{6}^{\mathrm{a}}\right]^{4-}(\mathrm{Q}=\mathrm{S}$ or Se $)$ is reported. However $\left[\mathrm{Mo}_{6} \mathrm{Br}_{6}{ }_{6} \mathrm{Q}_{2}^{\mathrm{i}}(\mathrm{CN})_{6}{ }_{6}\right]^{4-}(\mathrm{Q}=\mathrm{S}$ or Se $)$ and $\left[\operatorname{Re}_{6} \mathrm{Q}_{8}^{\mathrm{i}}(\mathrm{CN})_{6}^{\mathrm{a}}{ }_{6}\right]^{4-}$, which are both face capped units, are isoelectronic and they both carry a four minus charge. Consequently, the use of $\left[\mathrm{Mo}_{6} \mathrm{Br}_{6}^{\mathrm{i}} \mathrm{Q}_{2}^{\mathrm{i}}(\mathrm{CN})_{6}^{\mathrm{a}}\right]^{4-}$ should lead to compounds exhibiting architectures closer to those of $\operatorname{Re}_{6}$ compounds than those of $\mathrm{Nb}_{6}$ edge bridged-based compounds. Two compounds based on $\left[\mathrm{Mo}_{6} \mathrm{Br}_{6}^{\mathrm{i}}{ }_{6} \mathrm{Q}_{2}^{\mathrm{i}}(\mathrm{CN})_{6}^{\mathrm{a}}\right]^{4-}$ are already reported in the literature [29]. These are $\mathrm{Cs}_{0.4} \mathrm{~K}_{0.6}\left(\mathrm{Et}_{4} \mathrm{~N}\right)_{11}\left[\mathrm{Mo}_{6} \mathrm{Br}_{6} \mathrm{~S}_{2}(\mathrm{CN})_{6}\right]_{3} \quad$ and $\mathrm{Cs}_{0.5} \mathrm{~K}_{0.5}\left(\mathrm{Et}_{4} \mathrm{~N}\right)_{11}\left[\mathrm{Mo}_{6} \mathrm{Br}_{6} \mathrm{Se}_{2}(\mathrm{CN})_{6}\right]_{3}$ in which the alkali metals and $\left[\mathrm{Mo}_{6} \mathrm{Br}_{6}^{\mathrm{i}} \mathrm{Q}_{2}^{\mathrm{i}}(\mathrm{CN})_{6}^{\mathrm{a}}\right]^{4-}$ anions form a $\left\{\mathrm{M}\left[\mathrm{Mo}_{6} \mathrm{Br}_{6} \mathrm{Q}_{2}(\mathrm{CN})_{6}\right]_{3}\right\}^{11-}(\mathrm{M}=\mathrm{Cs}, \mathrm{K})$ framework with a topology related to that found in Super Prussian Blue Series [30-31], in particular cluster expanded Prussian Blue analogues [4-11]. In this frame, here we report the syntheses and crystal structures of two new compounds based on $\left[\mathrm{Mo}_{6} \mathrm{Br}_{6}{ }_{6} \mathrm{Se}_{2}^{\mathrm{i}}(\mathrm{CN})_{6}^{\mathrm{a}}\right]^{4-}$ cluster unit and $\mathrm{Zn}^{2+}$ or $\mathrm{Cu}^{2+}$ cations complexed by ethylenediamine namely $\left[\left\{\mathrm{Zn}\left(\mathrm{H}_{2} \mathrm{O}\right)(\mathrm{en})_{2}\right\}\left\{\mathrm{Zn}(\mathrm{en})_{2}\right\} \mathrm{Mo}_{6} \mathrm{Br}_{6} \mathrm{Se}_{2}(\mathrm{CN})_{6}\right] \cdot 2 \mathrm{H}_{2} \mathrm{O}$ (1) and $\left[\left\{\mathrm{Cu}\left(\mathrm{H}_{2} \mathrm{O}\right)(\mathrm{en})_{2}\right\}_{2} \mathrm{Mo}_{6} \mathrm{Br}_{6} \mathrm{Se}_{2}(\mathrm{CN})_{6}\right] \cdot 2 \mathrm{H}_{2} \mathrm{O}$ (2) (en = ethylenediamine). $\mathbf{1}$ is a bimetallic one dimensional coordination compound while 2 is a bimetallic $\mathrm{H}$ bonded three dimensional compound. Structural correlations with their Re analogues are discussed.

\section{EXPERIMENTAL}

Commercially available reagents and solvents were used without further purification. $\mathbf{1}$ and $\mathbf{2}$ have been obtained following a multi-step synthesis. Indeed, first the $\mathrm{MoBr}_{2}$ cluster compound is synthesized by placing Mo (Plansee) powder under $\mathrm{Br}_{2}$ gas flow according to a described procedure [32]. Then, $\mathrm{MoBr}_{2}, \mathrm{CsBr}$ (Alfa Aesar, 99.9\%), Mo (Plansee) and Se (Alfa Aesar, 99.999\%) are introduced in stoichiometric proportions in a vacuum sealed tube and heated at $900^{\circ} \mathrm{C}$ for two days to form $\mathrm{Cs}_{4}\left[\mathrm{Mo}_{6} \mathrm{Br}_{12} \mathrm{Se}_{2}\right]$ compound as previously described [29].

Elementary analyses for the heavy atoms have been done by Electron Dispersion Spectroscopy (EDS) with a scanning electron microscope JSM 7100F. Infrared red spectra were recorded on a Bruker Equinox 55 FTIR spectrometer on transmittance with $\mathrm{KBr}$ as reference.

$\mathrm{Cs}_{2} \mathbf{K}_{2}\left[\mathbf{M o}_{6} \mathbf{B r}_{6} \mathbf{S e}_{2}(\mathbf{C N})_{6}\right] .270 \mathrm{mg}$ of KCN (4.1 mmol) were dissolved in $20 \mathrm{~mL}$ of methanol in a Schlenk tube. Then, $1 \mathrm{~g}$ of $\mathrm{Cs}_{4}\left[\mathrm{Mo}_{6} \mathrm{Br}_{12} \mathrm{Se}_{2}\right]$ (0.45 mmol), prepared via solid state route at high temperature was added to the mixture and stirred $48 \mathrm{~h}$ under reflux at $55^{\circ} \mathrm{C}$. The resulting brown solution was filtered and methanol was evaporated almost to dryness. Then dicholoromethane was added to separate the excess of $\mathrm{KCN}$ and $\mathrm{KBr}$ by precipitation. This operation is repeated until there is no $\mathrm{KCN}$ and $\mathrm{KBr}$ anymore. Yield: $65 \%(0.5 \mathrm{~g}, 0.29 \mathrm{mmol})$.

$\operatorname{IR}\left(\mathrm{cm}^{-1}\right): 2113\left(v_{\mathrm{CN}}\right)$.

EDS analysis, heavy atoms \%: K, 11.2; Cs, 11.2; Mo, 33.2; $\mathrm{Br}$ 33.6; Se, 10.8. Calcd for $\mathrm{Cs}_{2} \mathrm{~K}_{2}\left[\mathrm{Mo}_{6} \mathrm{Br}_{6} \mathrm{Se}_{2}(\mathrm{CN})_{6}\right]$ : K, 11.1; Cs, 11.1; Mo, 33.3; Br, 33.3; Se, 11.1.

$\left[\left\{\mathrm{Zn}\left(\mathrm{H}_{2} \mathrm{O}\right)(\mathrm{en})_{2}\right\}\left\{\mathrm{Zn}(\mathrm{en})_{2}\right\}-\mathrm{Mo}_{6} \mathrm{~B}_{\mathrm{r} 6} \mathrm{Se}_{2}(\mathrm{CN})_{6}\right] .2 \mathrm{H}_{2} \mathrm{O}(1)$. In a narrow-diameter tube, a solution of $1.0 \mathrm{mg}(0.58 \mu \mathrm{mol})$ of $\mathrm{Cs}_{2} \mathrm{~K}_{2}\left[\mathrm{Mo}_{6} \mathrm{Br}_{6} \mathrm{Se}_{2}(\mathrm{CN})_{6}\right]$ in $0.5 \mathrm{~mL}$ of water was allowed to diffuse into a solution of $3.5 \mathrm{mg}$ of $\mathrm{Zn}\left[\mathrm{OOCCH}_{3}\right]_{2} \cdot 2 \mathrm{H}_{2} \mathrm{O}$ $(0.016 \mathrm{mmol}$ ) (Fluka Analytical, 99.5\%) in $0.5 \mathrm{~mL}$ of water to which $0.09 \mathrm{~mL}(1.35 \mathrm{mmol})$ of ethylenediamine (Alfa Aesar, 99\%) had been added. After 1 week dark red-brown crystals were obtained. Yield: 40\% (0.04 mg, $0.22 \mu \mathrm{mol})$.

IR $\left(\mathrm{cm}^{-1}\right)$ : 2109 and $2131\left(v_{\mathrm{CN}}\right)$.

EDS analysis, heavy atoms \%: Zn, $12.6 \mathrm{Mo}, 38.8$; Br, 36.9; Se, 11.7. calcd for $\left[\left\{\mathrm{Zn}\left(\mathrm{H}_{2} \mathrm{O}\right)(\mathrm{en})_{2}\right\}\left\{\mathrm{Zn}(\mathrm{en})_{2}\right\}-\right.$ $\left.\mathrm{Mo}_{6} \mathrm{~B}_{\mathrm{r} 6} \mathrm{Se}_{2}(\mathrm{CN})_{6}\right] .2 \mathrm{H}_{2} \mathrm{O}$ : Zn, 12.5; Mo, 37.5; Br, 37.5; Se, 12.5 .

$\left[\left\{\mathrm{Cu}\left(\mathrm{H}_{2} \mathrm{O}\right)(\mathrm{en})_{2}\right\}_{2} \mathrm{Mo}_{6} \mathrm{Br}_{6} \mathrm{Se}_{2}(\mathrm{CN})_{6}\right] .2 \mathrm{H}_{2} \mathrm{O}$ (2). In a narrowdiameter tube, a solution of $1.0 \mathrm{mg}(0.58 \mu \mathrm{mol})$ of $\mathrm{Cs}_{2} \mathrm{~K}_{2}\left[\mathrm{Mo}_{6} \mathrm{Br}_{6} \mathrm{Se}_{2}(\mathrm{CN})_{6}\right]$ in $0.5 \mathrm{~mL}$ of water was allowed to diffuse into a solution of $3.5 \mathrm{mg}$ of $\mathrm{CuCl}_{2} \cdot \mathrm{xH}_{2} \mathrm{O}$ (Aldrich) in $0.5 \mathrm{~mL}$ of ammonia (25\%) to which $0.09 \mathrm{~mL}$ (1.35 mmol) of ethylenediamine (Alfa Aesar, 99\%) had been added. After 3 weeks dark red-brown crystals were obtained.

$\mathrm{CuCl}_{2}$ and ethylenediamine were solubilized in ammonia for creating a competition between ammonia and ethylenediamine around $\mathrm{Cu}^{2+}$ cation in order to slow down the reaction because at first attempts an amorphous precipitate formed very quickly without formation of a crystalline phase. Yield: $30 \%$ (0.3 mg, $0.17 \mu \mathrm{mol})$.

$\operatorname{IR}\left(\mathrm{cm}^{-1}\right): 2104\left(v_{\mathrm{CN}}\right)$.

EDS analysis, heavy atoms \%: $\mathrm{Cu}, 13.3$; $\mathrm{Mo}, 37.8 ; \mathrm{Br}, 36.7$; Se, 12.2. calcd for $\left[\left\{\mathrm{Cu}\left(\mathrm{H}_{2} \mathrm{O}\right)(\mathrm{en})_{2}\right\}_{2} \mathrm{Mo}_{6} \mathrm{Br}_{6} \mathrm{Se}_{2}(\mathrm{CN})_{6}\right] \cdot 2 \mathrm{H}_{2} \mathrm{O}$ : $\mathrm{Cu}, 12.5$; Mo, 37.5; $\mathrm{Br}, 37.5$; Se, 12.5 .

Crystal structure determinations. Crystallographic data, details on data collections and refinement parameters of the crystal structures are summarized in Table 1 . Single-crystal $\mathrm{X}$-ray diffraction data were collected at $150 \mathrm{~K}$ on a D8 VENTURE Bruker AXS diffractometer and processed with the APEX 3 [33] program suite for $\mathbf{1}$. For 2, data were collected at room temperature on an APEX-II Bruker AXS diffractometer and processed with the APEX 2 [34] program suite. For both compounds the X-ray wavelength used was the $\mathrm{Mo}-\mathrm{K} \alpha(\lambda=0.71073 \AA)$. Frame integration and data reduction were carried out with the program SAINT [35]. The program SADABS [36] was then employed for multiscan-type absorption corrections. 
Table 1. Crystal data and structure refinement parameters for compounds $\mathbf{1}$ and $\mathbf{2 .}$

\begin{tabular}{|c|c|c|}
\hline & 1 & 2 \\
\hline Empirical formula & $\mathrm{C}_{14} \mathrm{H}_{38} \mathrm{Zn}_{2} \mathrm{~N}_{14} \mathrm{O}_{3} \mathrm{Mo}_{6} \mathrm{Br}_{6} \mathrm{Se}_{2}$ & $\mathrm{C}_{14} \mathrm{H}_{40} \mathrm{Cu}_{2} \mathrm{~N}_{14} \mathrm{O}_{4} \mathrm{Mo}_{6} \mathrm{Br}_{6} \mathrm{Se}_{2}$ \\
\hline Formula weight (g.mol ${ }^{-1}$ ) & 1786.28 & 1800.6 \\
\hline Crystal size $\left(\mathrm{mm}^{3}\right)$ & $0.14 \times 0.12 \times 0.06$ & $0.33 \times 0.15 \times 0.03$ \\
\hline Crystal color & brown & brown \\
\hline Crystal system & orthorhombic & monoclinic \\
\hline Space group & $P 2{ }_{1} 2_{1} 2_{1}(\underline{19})$ & $P 2_{1} / c(\underline{14})$ \\
\hline$a(\AA)$ & $10.675(1)$ & $9.4281(4)$ \\
\hline$b(\AA)$ & $15.073(2)$ & $11.0220(5)$ \\
\hline$c(\AA)$ & $25.812(4)$ & 21.1494(9) \\
\hline$\beta\left(^{\circ}\right)$ & & $100.053(2)$ \\
\hline Volume $\left(\AA^{3}\right)$ & 4153(1) & $2164.0(2)$ \\
\hline $\mathrm{Z}$ & 4 & 2 \\
\hline$\rho($ calcd. $)\left(\right.$ g.cm $\left.{ }^{-3}\right)$ & 2.86 & 2.76 \\
\hline$\mu\left(\mathrm{mm}^{-1}\right)$ & 10.449 & 9.904 \\
\hline$F(000)$ & 3304 & 1668 \\
\hline$\lambda(\AA)$ & 0.71073 & 0.71073 \\
\hline $\mathrm{T}(\mathrm{K})$ & 150 & 296 \\
\hline $2 \Theta$ range $\left({ }^{\circ}\right)$ & 2.34 to 35.43 & 2.67 to 28.3 \\
\hline Collected reflections & 48311 & 9883 \\
\hline Independent reflections & 26337 & 5818 \\
\hline $\begin{array}{l}\text { Observed reflections } \\
{[I>2 \sigma(I)]}\end{array}$ & 12243 & 3594 \\
\hline Data / restrains & 26337 / 0 / 413 & $5818 / 0 / 217$ \\
\hline / refined parameters & & \\
\hline Goodness-of-fit on $\mathrm{F}^{2}$ & 0.959 & 1.023 \\
\hline $\mathrm{R}_{1}, \omega \mathrm{R}_{2}$ & $0.064,0.125$ & $0.044,0.108$ \\
\hline $\mathrm{R}_{1}, \omega \mathrm{R}_{2}$ (all data) & $0.185,0.153$ & $0.094,0.121$ \\
\hline Large. diff. peak and hole (e. $\AA^{-3}$ ) & $2.513,-1.715$ & $1.209,-1.202$ \\
\hline
\end{tabular}

The structure was determined by direct method using the SHELXT program [37], and refined with full-matrix leastsquare methods based on $\mathrm{F}^{2}$ (SHELXL-2014) [38] with the aid of the WinGX plateform [39]. The final refinements included anisotropic displacement parameters for the nonhydrogen atoms. For both compounds the positions of the hydrogen atoms on the ethylenediamine groups were idealized by HFIX command.

\section{RESULTS AND DISCUSSIONS}

Synthesis. $\mathrm{K}_{2} \mathrm{Cs}_{2}\left[\mathrm{Mo}_{6} \mathrm{Br}_{6} \mathrm{Se}_{2}(\mathrm{CN})_{6}\right]$ was synthetized and used as precursor because of its solubility and its stability in water contrary to $\mathrm{Cs}_{0.5} \mathrm{~K}_{0.5}\left(\mathrm{Et}_{4} \mathrm{~N}\right)_{11}\left[\mathrm{Mo}_{6} \mathrm{Br}_{6} \mathrm{Se}_{2}(\mathrm{CN})_{6}\right]_{3} .16 \mathrm{H}_{2} \mathrm{O}$ [29] which is precipitates in water at room temperature. Both $\mathbf{1}$ and $\mathbf{2}$ have been obtained as single-crystals.

Crystal structures. 1 crystallizes in the orthorhombic space group $P 2_{1} 2_{1} 2_{1}$ with refined unit-cell parameters $a=$ 10.675(1) $\AA, b=15.073(2) \AA, c=25.812(4) \AA$ and $V=$ 4153(1) $\AA^{3} .2$ crystallizes in the monoclinic space group
$P 2_{1} / c$ with refined parameters $a=9.4281(4) \AA, b=$ 11.0220(5) $\AA, c=21.1494(9) \AA, \beta=100.053(2)^{\circ}$ and $V=2164.0(2) \AA^{3}$. All atoms fully occupy general Wyckoff positions [i.e. $4 a$ and $4 e$ for $\mathbf{1}$ and 2 , respectively (Table 2)] excepted face capping positions, randomly occupied by $\mathrm{Br}$ (75\%) and Se (25\%) as discussed below.

Both 1 and 2 are built up from a $\left[\mathrm{Mo}_{6} \mathrm{Br}_{6}^{\mathrm{i}} \mathrm{Se}_{2}^{\mathrm{i}}(\mathrm{CN})_{6}^{\mathrm{a}}\right]^{4-}$ cluster unit where the face-capping positions of the octahedral cluster are randomly occupied by six bromine and two selenium atoms. The six apical positions are fully occupied by cyanide ligands as depicted in Figure 1. The presence of both bromine and selenium elements could not be distinguished by X-ray diffraction analyses but has been clearly observed and quantified by EDS analyses. Thus, the value of the $\mathrm{Br} / \mathrm{Se}$ ratio was fixed at 0.75:0.25 during the refinement according to chemical analyses. The average MoMo distances are 2.639(1) and 2.642(1) $\AA$ in $\mathbf{1}$ and 2, respectively. The Mo- $\mathrm{L}(\mathrm{L}=\mathrm{Br} / \mathrm{Se})$ distances range from 2.572(1) to 2.629(1) Å in 1 and from 2.5590(9) to 2.6148(9) $\AA$ in 2. 
Table 2. Atomic coordinates, site occupancy and equivalent isotropic displacement $\left(\AA^{2}\right)$ parameters refined at $150 \mathrm{~K}$ and at room temperature for compound $\mathbf{1}$ and $\mathbf{2}$, respectively.

\begin{tabular}{|c|c|c|c|c|c|c|}
\hline \multicolumn{7}{|c|}{$\left[\left\{\mathrm{Zn}\left(\mathrm{H}_{2} \mathrm{O}\right)(\mathrm{en})_{2}\right\}\left\{\mathrm{Zn}(\mathrm{en})_{2}\right\}-\mathrm{Mo}_{6} \mathrm{~B}_{\mathrm{r} 6} \mathrm{Se}_{2}(\mathrm{CN})_{6}\right] \cdot 2 \mathrm{H}_{2} \mathrm{O}(\mathbf{1})$} \\
\hline Atom & Site & $\mathrm{x}$ & $\mathrm{y}$ & $\mathrm{z}$ & Occ. & $\mathrm{U}(\mathrm{eq})$ \\
\hline Mo1 & $4 a$ & $0.3872(1)$ & $0.3871(1)$ & $0.3371(1)$ & 1 & $0.017(1)$ \\
\hline Mo2 & $4 a$ & $0.5834(1)$ & $0.4596(1)$ & $0.2901(1)$ & 1 & $0.018(1)$ \\
\hline Mo3 & $4 a$ & $-0.3696(1)$ & $0.0467(1)$ & $0.2028(1)$ & 1 & $0.019(1)$ \\
\hline Mo4 & $4 a$ & $0.5653(1)$ & $0.5979(1)$ & $0.3528(1)$ & 1 & $0.020(1)$ \\
\hline Mo5 & $4 a$ & $0.5841(1)$ & 0.4369(1) & 0.3918(1) & 1 & $0.019(1)$ \\
\hline Mo6 & $4 a$ & $0.3705(1)$ & $0.5248(1)$ & $0.3992(1)$ & 1 & $0.019(1)$ \\
\hline Br1 & $4 a$ & 0.5997(1) & $0.3011(1)$ & $0.3289(1)$ & 0.75 & $0.023(1)$ \\
\hline Se1 & $4 a$ & 0.5997(1) & $0.3011(1)$ & $0.3289(1)$ & 0.25 & $0.023(1)$ \\
\hline Br2 & $4 a$ & $0.3873(1)$ & 0.4084(1) & $0.2369(1)$ & 0.75 & $0.024(1)$ \\
\hline Se2 & $4 a$ & $0.3873(1)$ & $0.4084(1)$ & $0.2369(1)$ & 0.25 & $0.024(1)$ \\
\hline $\mathrm{Br} 3$ & $4 a$ & $-0.1788(1)$ & $-0.0237(1)$ & $0.1561(1)$ & 0.75 & $0.028(1)$ \\
\hline Se3 & $4 a$ & $-0.1788(1)$ & $-0.0237(1)$ & $0.1561(1)$ & 0.25 & $0.028(1)$ \\
\hline Br4 & $4 a$ & $-0.3705(1)$ & $0.1827(1)$ & $0.1417(1)$ & 0.75 & $0.028(1)$ \\
\hline Se4 & $4 a$ & $-0.3705(1)$ & 0.1827(1) & $0.1417(1)$ & 0.25 & $0.028(1)$ \\
\hline Br5 & $4 a$ & $0.5637(1)$ & 0.6197(1) & $0.2525(1)$ & 0.75 & $0.026(1)$ \\
\hline Se5 & $4 a$ & $0.5637(1)$ & 0.6197(1) & $0.2525(1)$ & 0.25 & $0.026(1)$ \\
\hline Br6 & $4 a$ & $0.7726(1)$ & $0.5082(1)$ & $0.3450(1)$ & 0.75 & $0.029(1)$ \\
\hline Se6 & $4 a$ & $0.7726(1)$ & $0.5082(1)$ & $0.3450(1)$ & 0.25 & $0.029(1)$ \\
\hline Br7 & $4 a$ & $0.5652(1)$ & 0.5738(1) & $0.4521(1)$ & 0.75 & $0.028(1)$ \\
\hline Se7 & $4 a$ & $0.5652(1)$ & 0.5738(1) & $0.4521(1)$ & 0.25 & $0.028(1)$ \\
\hline Br8 & $4 a$ & $0.3888(1)$ & $0.3655(1)$ & $0.4368(1)$ & 0.75 & $0.027(1)$ \\
\hline Se8 & $4 a$ & $0.3888(1)$ & $0.3655(1)$ & $0.4368(1)$ & 0.25 & $0.027(1)$ \\
\hline C1 & $4 a$ & $0.2750(9)$ & $0.2676(7)$ & $0.3297(4)$ & 1 & $0.024(2)$ \\
\hline C2 & $4 a$ & 0.709(1) & $0.4226(8)$ & $0.2236(5)$ & 1 & $0.032(2)$ \\
\hline C3 & $4 a$ & $-0.247(1)$ & $0.1093(7)$ & $0.2617(5)$ & 1 & $0.030(2)$ \\
\hline C4 & $4 a$ & $0.663(1)$ & $0.7257(7)$ & $0.3644(5)$ & 1 & $0.030(2)$ \\
\hline C5 & $4 a$ & $0.710(1)$ & $0.3704(7)$ & $0.4487(5)$ & 1 & $0.030(2)$ \\
\hline C6 & $4 a$ & $0.241(1)$ & $0.5568(7)$ & $0.4659(5)$ & 1 & $0.030(2)$ \\
\hline C7 & $4 a$ & $0.029(3)$ & $0.110(1)$ & $0.4190(8)$ & 1 & $0.133(8)$ \\
\hline C8 & $4 a$ & $-0.026(3)$ & $0.190(1)$ & $0.4244(8)$ & 1 & $0.33(8)$ \\
\hline C9 & $4 a$ & $0.017(2)$ & $0.203(1)$ & $0.2016(7)$ & 1 & $0.083(6)$ \\
\hline C10 & $4 a$ & $0.048(2)$ & $0.293(1)$ & $0.2215(6)$ & 1 & $0.072(6)$ \\
\hline C11 & $4 a$ & $0.033(2)$ & $0.375(1)$ & $0.5774(8)$ & 1 & $0.105(6)$ \\
\hline C12 & $4 a$ & $0.090(2)$ & $0.438(1)$ & $0.6111(9)$ & 1 & $0.105(6)$ \\
\hline C13 & $4 a$ & $-0.058(2)$ & $0.674(1)$ & $0.4457(7)$ & 1 & $0.081(6)$ \\
\hline C14 & $4 a$ & $-0.115(2)$ & $0.7143(9)$ & $0.4965(7)$ & 1 & $0.067(5)$ \\
\hline N1 & $4 a$ & $0.205(1)$ & $0.2122(7)$ & $0.3272(4)$ & 1 & $0.041(3)$ \\
\hline N2 & $4 a$ & $0.768(1)$ & $0.4105(8)$ & $0.1892(5)$ & 1 & $0.054(3)$ \\
\hline N3 & $4 a$ & $-0.1857(9)$ & $0.1395(6)$ & $0.2922(4)$ & 1 & $0.029(2)$ \\
\hline N4 & $4 a$ & $0.709(1)$ & $0.7925(7)$ & $0.3723(4)$ & 1 & $0.041(3)$ \\
\hline N5 & $4 a$ & $0.775(1)$ & $0.3414(7)$ & $0.4767(5)$ & 1 & $0.049(3)$ \\
\hline N6 & $4 a$ & $0.190(1)$ & $0.5697(7)$ & $0.5008(4)$ & 1 & $0.044(3)$ \\
\hline N7 & $4 a$ & 0.031(1) & $0.0737(7)$ & $0.3643(6)$ & 1 & $0.058(4)$ \\
\hline N8 & $4 a$ & $-0.036(1)$ & $0.2527(8)$ & $0.3857(5)$ & 1 & $0.052(3)$ \\
\hline N9 & $4 a$ & $-0.017(1)$ & $0.3048(8)$ & $0.2706(4)$ & 1 & $0.046(3)$ \\
\hline N10 & $4 a$ & $0.055(1)$ & 0.132 (1) & $0.2410(5)$ & 1 & $0.063(4)$ \\
\hline N11 & $4 a$ & $0.029(1)$ & $0.4065(8)$ & $0.5199(5)$ & 1 & $0.058(4)$ \\
\hline N12 & $4 a$ & $0.037(1)$ & $0.5241(9)$ & $0.6087(4)$ & 1 & $0.061(4)$ \\
\hline N13 & $4 a$ & $-0.062(1)$ & $0.5732(8)$ & $0.4518(5)$ & 1 & $0.055(3)$ \\
\hline N14 & $4 a$ & $-0.049(1)$ & $0.6816(8)$ & $0.5399(5)$ & 1 & $0.062(4)$ \\
\hline O1 & $4 a$ & $-0.2024(9)$ & $0.5036(8)$ & $0.5501(5)$ & 1 & $0.066(3)$ \\
\hline $\mathrm{O} 2$ & $4 a$ & $-0.3539(9)$ & $0.6308(6)$ & $0.5878(4)$ & 1 & $0.046(2)$ \\
\hline O3 & $4 a$ & $0.8818(9)$ & $0.9144(6)$ & $0.4102(4)$ & 1 & $0.046(2)$ \\
\hline Zn1 & $4 a$ & $-0.0015(1)$ & $0.1819(1)$ & $0.3142(1)$ & 1 & $0.028(1)$ \\
\hline Zn2 & $4 a$ & $-0.0080(1)$ & $0.5452(1)$ & $0.5296(1)$ & 1 & $0.034(1)$ \\
\hline
\end{tabular}




\begin{tabular}{|c|c|c|c|c|c|c|}
\hline \multicolumn{7}{|c|}{$\left[\left\{\mathrm{Cu}\left(\mathrm{H}_{2} \mathrm{O}\right)(\mathrm{en})_{2}\right\}_{2} \mathrm{Mo}_{6} \mathrm{Br}_{6} \mathrm{Se}_{2}(\mathrm{CN})_{6}\right] \cdot 2 \mathrm{H}_{2} \mathrm{O}(2)$} \\
\hline Atom & Site & $\mathrm{x}$ & $\mathrm{y}$ & $\mathrm{Z}$ & Occ. & $\mathrm{U}(\mathrm{eq})$ \\
\hline Mo1 & $4 e$ & $0.4392(1)$ & $0.4421(1)$ & $0.5740(1)$ & 1 & $0.024(1)$ \\
\hline Mo2 & $4 e$ & $0.5908(1)$ & $0.3512(1)$ & $0.4916(1)$ & 1 & $0.024(1)$ \\
\hline Mo3 & $4 e$ & $0.6685(1)$ & 0.5588(1) & $0.5502(1)$ & 1 & $0.024(1)$ \\
\hline $\mathrm{Br} 1$ & $4 e$ & $0.3638(1)$ & $0.2377(1)$ & $0.5149(1)$ & 0.75 & $0.036(1)$ \\
\hline Se1 & $4 e$ & $0.3638(1)$ & $0.2377(1)$ & $0.5149(1)$ & 0.25 & $0.036(1)$ \\
\hline $\mathrm{Br} 2$ & $4 e$ & $0.8164(1)$ & $0.4676(1)$ & $0.4678(1)$ & 0.75 & $0.037(1)$ \\
\hline Se2 & $4 e$ & $0.8164(1)$ & $0.4676(1)$ & $0.4678(1)$ & 0.25 & $0.037(1)$ \\
\hline $\mathrm{Br} 3$ & $4 e$ & $0.5164(1)$ & $0.6473(1)$ & $0.6308(1)$ & 0.75 & $0.034(1)$ \\
\hline Se3 & $4 e$ & $0.5164(1)$ & $0.6473(1)$ & $0.6308(1)$ & 0.25 & $0.034(1)$ \\
\hline $\mathrm{Br} 4$ & $4 e$ & $0.6925(1)$ & $0.3581(1)$ & $0.6127(1)$ & 0.75 & $0.039(1)$ \\
\hline Se4 & $4 e$ & $0.6925(1)$ & $0.3581(1)$ & $0.6127(1)$ & 0.25 & $0.039(1)$ \\
\hline C1 & $4 e$ & $0.3658(8)$ & $0.3684(7)$ & $0.6605(4)$ & 1 & $0.040(2)$ \\
\hline C2 & $4 e$ & $0.6980(9)$ & $0.1734(7)$ & $0.4839(4)$ & 1 & $0.040(2)$ \\
\hline C3 & $4 e$ & $0.8657(7)$ & $0.6238(6)$ & $0.6125(4)$ & 1 & $0.031(2)$ \\
\hline $\mathrm{C} 4$ & $4 e$ & $0.705(1)$ & $1.008(1)$ & $0.6403(6)$ & 1 & $0.091(4)$ \\
\hline C5 & $4 e$ & $0.7338(9)$ & $1.0707(9)$ & $0.6984(6)$ & 1 & $0.068(3)$ \\
\hline C6 & $4 e$ & $1.279(1)$ & $0.8858(9)$ & $0.6953(5)$ & 1 & $0.066(3)$ \\
\hline C7 & $4 e$ & $1.3213(9)$ & $1.0134(8)$ & $0.5855(4)$ & 1 & $0.056(2)$ \\
\hline N1 & $4 e$ & $0.3186(9)$ & $0.3259(7)$ & $0.7019(4)$ & 1 & $0.063(2)$ \\
\hline N2 & $4 e$ & $0.7583(8)$ & $0.0831(6)$ & $0.4806(3)$ & 1 & $0.056(2)$ \\
\hline N3 & $4 e$ & $0.9638(7)$ & $0.6511(6)$ & $0.6477(3)$ & 1 & $0.049(2)$ \\
\hline N4 & $4 e$ & $0.8269(7)$ & $0.9392(7)$ & $0.6262(4)$ & 1 & $0.077(3)$ \\
\hline N5 & $4 e$ & $0.8810(7)$ & $1.1233(6)$ & $0.7101(3)$ & 1 & $0.046(2)$ \\
\hline N6 & $4 e$ & $1.11413(7)$ & $0.8827(6)$ & $0.6494(4)$ & 1 & $0.057(2)$ \\
\hline N7 & $4 e$ & $1.1978(6)$ & $1.0760(6)$ & $0.7267(3)$ & 1 & $0.042(2)$ \\
\hline $\mathrm{O} 1$ & $4 e$ & $1.0470(8)$ & $1.1485(8)$ & $0.5855(4)$ & 1 & $0.104(3)$ \\
\hline $\mathrm{O} 2$ & $4 e$ & $0.0225(7)$ & $0.3780(7)$ & $0.7057(4)$ & 1 & $0.093(3)$ \\
\hline Cu1 & $4 e$ & $1.0146(1)$ & $1.0182(1)$ & $0.6694(1)$ & 1 & $0.045(1)$ \\
\hline
\end{tabular}

These distances are within the range observed in $\mathrm{Cs}_{0.5} \mathrm{~K}_{0.5}\left(\mathrm{Et}_{4} \mathrm{~N}\right)_{11}\left[\mathrm{Mo}_{6} \mathrm{Br}_{6} \mathrm{Se}_{2}(\mathrm{CN})_{6}\right]_{3}$ [29] which is built up from the same $\left[\mathrm{Mo}_{6} \mathrm{Br}_{6}{ }_{6} \mathrm{Si}_{2}{ }_{2}(\mathrm{CN})_{6}^{\mathrm{a}}\right]^{4-}$ cluster unit. Relevant selected bond lengths and angles are listed in Table 3.

The structure of $\mathbf{1}$ can be depicted by the association of one $\left[\mathrm{Mo}_{6} \mathrm{Br}_{6}{ }_{6}^{\mathrm{i}} \mathrm{Se}_{2}^{\mathrm{i}}(\mathrm{CN})_{6}^{\mathrm{a}}\right]^{4-}$ cluster unit with one $\left[\mathrm{Zn}\left(\mathrm{H}_{2} \mathrm{O}\right) \mathrm{en}_{2}\right]^{2+}$ and one $\left[\mathrm{Zn}(\mathrm{en})_{2}\right]^{2+}$ groups. The $\left[\mathrm{Zn}\left(\mathrm{H}_{2} \mathrm{O}\right) \mathrm{en}_{2}\right]^{2+}$ groups and $\left[\mathrm{Mo}_{6} \mathrm{Br}_{6}{ }_{6}^{\mathrm{i}} \mathrm{Se}_{2}^{\mathrm{i}}(\mathrm{CN})_{6}^{\mathrm{a}}\right]^{4-}$ cluster units form $\left[\mathrm{Mo}_{6} \mathrm{Br}_{6} \mathrm{Se}_{2}(\mathrm{CN})_{5}^{\mathrm{a}} \mathrm{CN}-\mathrm{Zn}\left(\mathrm{H}_{2} \mathrm{O}\right) \mathrm{en}_{2}\right]^{2-} \quad$ hetero-metallic complexes via C6N6-Zn2 bonds as represented in Figure 2. The latter are then interconnected through $\left[\mathrm{Zn}(\mathrm{en})_{2}\right]^{2+}$ groups via $\mathrm{C} 1 \mathrm{~N} 1-\mathrm{Zn} 1$ and $\mathrm{C} 3 \mathrm{~N} 3-\mathrm{Zn} 1$ bonds to build infinite polymeric zigzag chains that spread along the $b$ axis of the unit cell as depicted in Figure 3. The infinite chains are stacked along the $c$ axis according to an $\mathrm{ABAB}$ stacking type. In the infrared spectra, two bands at 2109 and $2131 \mathrm{~cm}^{-1}$ can be assigned to the bridging and terminal CN ligands, respectively.

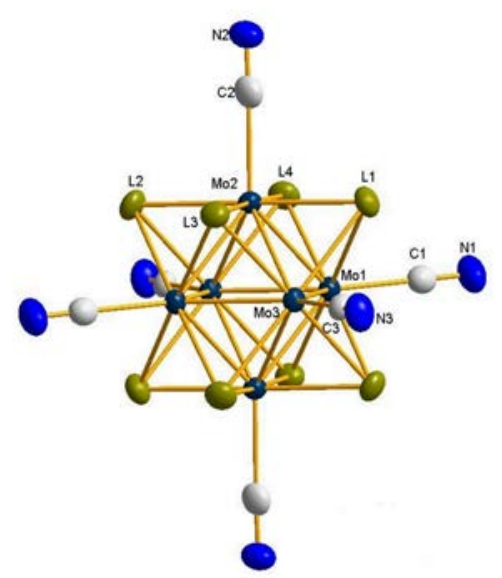

Figure 1. Representation of the $\left[\mathrm{Mo}_{6} \mathrm{Br}_{6}{ }_{6} \mathrm{Se}_{2}^{\mathrm{i}}(\mathrm{CN})_{6}^{\mathrm{a}}\right]^{4-}$ cluster unit in 2. L represents the $\mathrm{Br} / \mathrm{Se}$ inner ligands. Displacement ellipsoids are shown at the $50 \%$ probability level. 
Table 3. Selected bond lengths $(\AA)$ and angles $\left(^{\circ}\right)$ for compounds $\mathbf{1}, 2$ and $\mathrm{Cs}_{0.5} \mathrm{~K}_{0.5}\left(\mathrm{Et}_{4} \mathrm{~N}\right)_{11}\left[\mathrm{Mo}_{6} \mathrm{Br}_{6} \mathrm{Se}_{2}(\mathrm{CN})_{6}\right]_{3}$.

\begin{tabular}{|c|c|c|c|c|c|}
\hline \multicolumn{6}{|l|}{1} \\
\hline Mo1-Mo3 $^{\# 1}$ & $2.624(1)$ & Mo6-L3 $^{\# 1}$ & $2.599(1)$ & Mo1-C1-N1 & 171(1) \\
\hline Mo1-Mo6 & $2.628(1)$ & Mo6-L7 & $2.595(1)$ & Mo3-C3-N3 & 178(1) \\
\hline Mo2-Mo5 & $2.647(1)$ & Mo1-C1 & $2.17(1)$ & Mo5-C5-N5 & 177(1) \\
\hline Mo3-Mo1 & 2.624(1) & Mo2-C2 & $2.25(1)$ & Mo6-C6-N6 & 177(1) \\
\hline Mo3-Mo2 $2^{\# 2}$ & $2.640(1)$ & Mo3-C3 & $2.22(1)$ & C1-N1-Zn1 & $144(1)$ \\
\hline Mo4-Mo5 & $2.636(1)$ & Mo5-C5 & $2.23(1)$ & N1-Zn1-N3 & $170.8(4)$ \\
\hline Mo4-Mo6 & 2.641(1) & Mo6-C6 & $2.20(1)$ & C6-N6-Zn2 & $140(1)$ \\
\hline Mo5-Mo6 & 2.644(1) & C1-N1 & $1.13(1)$ & N6-Zn2-O1 & $171.5(4)$ \\
\hline Mo1-L1 & $2.622(1)$ & C2-N2 & $1.11(2)$ & & \\
\hline Mo1-L2 & $2.605(1)$ & C3-N3 & 1.12(1) & & \\
\hline Mo1-L3 & $2.605(1)$ & C4-N4 & $1.14(1)$ & & \\
\hline Mo2-L5 & $2.609(1)$ & Zn1-N3 & $2.14(1)$ & & \\
\hline Mo2-L6 & $2.572(1)$ & Zn1-N7 & 2.11(1) & & \\
\hline Mo3-L2 ${ }^{\# 2}$ & $2.608(1)$ & Zn1-N8 & $2.16(1)$ & & \\
\hline Mo3-L3 & 2.594(1) & Zn1-N9 & $2.18(1)$ & & \\
\hline Mo3-L4 & 2.594(1) & Zn1-N10 & $2.12(1)$ & & \\
\hline Mo3-L5 & $2.614(1)$ & Zn2-N6 & $2.27(1)$ & & \\
\hline Mo4-L4 ${ }^{\# 1}$ & 2.629(1) & Zn2-N11 & $2.14(1)$ & & \\
\hline Mo4-L5 & $2.609(2)$ & Zn2-N12 & $2.12(1)$ & & \\
\hline Mo4-L6 & 2.601(1) & Zn2-N13 & $2.13(1)$ & & \\
\hline Mo4-L7 & $2.588(2)$ & Zn2-N14 & $2.12(1)$ & & \\
\hline Mo5-L1 & 2.618(1) & Zn2-O1 & $2.23(1)$ & & \\
\hline Mo2-Mo3 & $2.6445(8)$ & C1-N1 & $1.150(9)$ & & \\
\hline Mo2-Mo3 ${ }^{\# 3}$ & $2.6440(8)$ & C2-N2 & $1.155(9)$ & & \\
\hline Mo1-L1 & 2.6135(9) & C3-N3 & 1.123(9) & & \\
\hline Mo1-L2 $2^{\# 3}$ & $2.6148(9)$ & Cu1-N4 & $2.039(7)$ & & \\
\hline Mo1-L3 & $2.6044(9)$ & Cu1-N5 & $2.012(6)$ & & \\
\hline Mo1-L4 & $2.5590(9)$ & Cu1-N6 & $2.003(7)$ & & \\
\hline Mo2-L1 & 2.5990(9) & Cu1-N7 & $2.031(6)$ & & \\
\hline Mo2-L2 & 2.6069(9) & Cu1-O1 & $2.343(7)$ & & \\
\hline Mo2-L3 ${ }^{\# 3}$ & 2.6102(9) & & & & \\
\hline Mo2-L4 & $2.575(1)$ & & & & \\
\hline Mo3-L1 ${ }^{\# 3}$ & $2.6224(9)$ & & & & \\
\hline Mo3-L2 & 2.6157(9) & & & & \\
\hline Mo3-L3 & $2.6018(9)$ & & & & \\
\hline Mo3-L4 & $2.5664(9)$ & & & & \\
\hline \multicolumn{6}{|c|}{$\mathrm{Cs}_{0.5} \mathrm{~K}_{0.5}\left(\mathrm{Et}_{4} \mathrm{~N}\right)_{11}\left[\mathrm{Mo}_{6} \mathrm{Br}_{6} \mathrm{Se}_{2}(\mathrm{CN})_{6}\right]_{3}$} \\
\hline Mo1-Mo2 & $2.6532(8)$ & Mo2-C2 & $2.22(1)$ & & \\
\hline Mo2-Mo2 & $2.647(1)$ & C1-N1 & $1.11(2)$ & & \\
\hline Mo1-L1 & $2.6006(6)$ & C2-N2 & 1.12(1) & & \\
\hline Mo2-L1 & $2.6042(5)$ & Mo1-C1-N1 & 180. & & \\
\hline Mo1-C1 & $2.23(1)$ & Mo2-C2-N2 & $180.0(8)$ & & \\
\hline
\end{tabular}

Symmetry transformations used to generate equivalent atoms:

$\# 1-x, y+1 / 2,-z+1 / 2 \quad \# 2-x, y-1 / 2,-z+1 / 2 \quad \# 3-x+1,-y+1,-z+1$. 


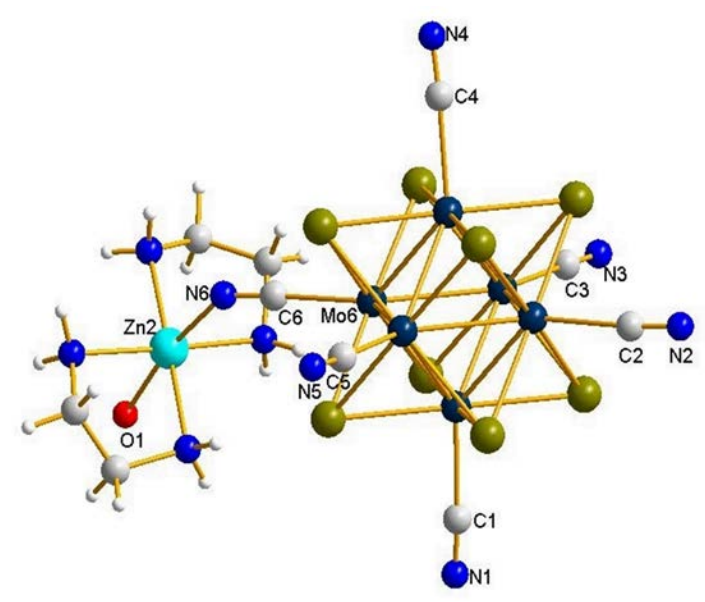

Figure 2. Representation of $\left[\mathrm{Mo}_{6} \mathrm{Br}_{6} \mathrm{Se}_{2}(\mathrm{CN})^{\mathrm{a}}{ }_{5} \mathrm{CN}\right.$ $\left.\mathrm{Zn}\left(\mathrm{H}_{2} \mathrm{O}\right) \mathrm{en}_{2}\right]^{2-}$ hetero-metallic complex in $\mathbf{1}$.

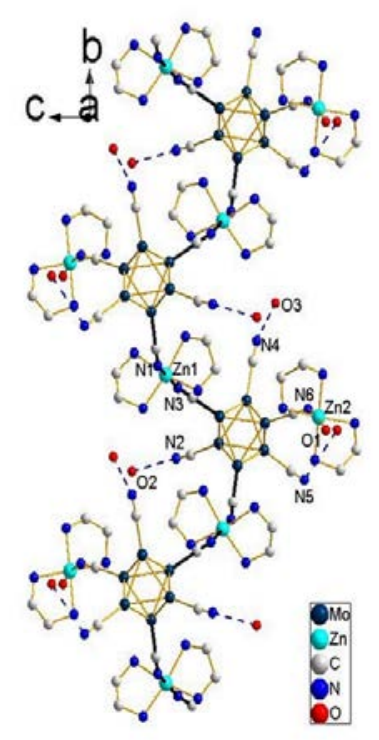

Figure 3. Representation of a one-dimensional polymeric chain in $\mathbf{1}$. Dashed lines represent hydrogen bonds between free cyanides ligands and water molecules. The inner ligands and the $\mathrm{H}$ atoms on the ethylenediamine groups have been omitted for clarity.

Terminal cyanides ligands (i.e. C2N2, C4N4 and C5N5) are involved in weak interactions with (i) crystallization water molecules, (ii) water molecule of the $\left[\mathrm{Zn}\left(\mathrm{H}_{2} \mathrm{O}\right) \mathrm{en}_{2}\right]^{2+}$ groups and (iii) nitrogen atoms of an ethylenediamine group (N2O2cw 2.8352(4); N4-O3cw 2.7943(2); N4-N12 3.36(2); N5O1w 3.0912(3); N5-N11 3.09(2) Å). Beyond these interactions, the inter chains structural cohesion is also ensured by other hydrogen bonds between nitrogen atom of the ethylenediamine groups and crystallization water molecule (N7-O3cw 3.12(2); O3cw-N6 3.0922(3) Å), between nitrogen atoms of the ethylenediamine groups and inner ligands (N13-L6 3.42(1); N14-L4 3.50(1) A), between crystallization water molecule and the water molecule of the $\left[\mathrm{Zn}\left(\mathrm{H}_{2} \mathrm{O}\right) \mathrm{en}_{2}\right]^{2+}$ groups $(\mathrm{O} 1-\mathrm{O} 2 \mathrm{cw} 2.7092(2) \AA)$ and between crystallization water molecules (O2cw-O3cw 2.9091(4) $\AA$ ). The intra chain structural cohesion is ensured by coordination bonds between the $\left[\mathrm{Mo}_{6} \mathrm{Br}_{6}^{\mathrm{i}} \mathrm{Se}_{2}^{\mathrm{i}}(\mathrm{CN})_{6}^{\mathrm{a}}\right]^{4-}$ cluster unit and $\mathrm{Zn}(\mathrm{en})_{2}$ groups. All the hydrogen bonds are listed in Table 4.

The structure of $\mathbf{1}$ is very similar to that of $\left[\left\{\mathrm{Zn}\left(\mathrm{H}_{2} \mathrm{O}\right)(\mathrm{en})_{2}\right\}\left\{\mathrm{Zn}(\mathrm{en})_{2}\right\}-\mathrm{Re}_{6} \mathrm{Te}_{8}(\mathrm{CN})_{6}\right] \cdot 3 \mathrm{H}_{2} \mathrm{O}$ (a) [22-23] and to that of $\left[\left\{\mathrm{Mn}\left(\mathrm{H}_{2} \mathrm{O}\right)(\mathrm{en})_{2}\right\}\left\{\mathrm{Mn}(\mathrm{en})_{2}\right\}-\right.$ $\left.\mathrm{Re}_{6} \mathrm{Te}_{8}(\mathrm{CN})_{6}\right] \cdot 3 \mathrm{H}_{2} \mathrm{O}$ (b) [40] which both crystallize in the monoclinic system, space group $P 2_{1} / n$ with refined parameters $a=10.7689(6) \AA, b=16.5594(9) \AA, c=$ 24.3631(14) $\AA, \beta=92.070(1)^{\circ}, V=4341.8(4) \AA^{3}$ and $a=$ 10.7940(1) $\AA, b=16.8011(3) \AA, c=24.6467(5) \AA, \beta=$ 92.055(1) ${ }^{\circ}, V=4466.8(1) \AA^{3}$ for $\mathbf{a}$ and $\mathbf{b}$, respectively. The loss of symmetry from orthorhombic to monoclinic system is probably only due to the additional crystallization water molecule in the lattice because the size of both $\left[\mathrm{Mo}_{6} \mathrm{Br}_{6}{ }_{6}^{\mathrm{i}} \mathrm{Se}_{2}^{\mathrm{i}}(\mathrm{CN})_{6}^{\mathrm{a}}\right]^{4-}$ and $\left[\mathrm{Re}_{6} \mathrm{Te}_{8}^{\mathrm{i}}(\mathrm{CN})_{6}^{\mathrm{a}}{ }_{6}\right]^{4-}$ cluster units are close (approximatively 10.40 and $10.35 \AA$ in diameter, respectively, according to a spherical shape model). In the $\left[\mathrm{M}_{6} \mathrm{X}_{8}^{\mathrm{i}}(\mathrm{CN})_{5}{ }_{5} \mathrm{CN}-\mathrm{Zn}\left(\mathrm{H}_{2} \mathrm{O}\right) \mathrm{en}_{2}\right]^{2-}$ hetero-metallic complexes $(\mathrm{M}=\mathrm{Mo}, \mathrm{Re} ; \mathrm{X}=\mathrm{Br}$, Se, Te), the $\mathrm{Zn}-\mathrm{N}-(-\mathrm{C}-\mathrm{M})$ distance in $\mathbf{1}$ is shorter than that in a [2.27(1) versus 2.339(6) $\AA$ ] while it is equivalent to the Mn-N(-C-Re) [2.23(1) versus 2.26(2) $\AA$ ] distance in $\mathbf{b}$. The same trend is found for $\mathrm{M}^{2+}-\mathrm{OH}_{2}$ distances $[2.23(1), 2.134(6)$ and 2.20(1) $\AA$ for $\mathbf{1}$, $\mathbf{a}$ and $\mathbf{b}$, respectively]. The average $\mathrm{M}^{2+}-\mathrm{N}(-\mathrm{C}-\mathrm{M})$ distances involved in $\mathrm{M}^{2+}(\mathrm{en})_{2}$ bridges are 2.21(1), 2.216(6) and 2.24(1) $\AA$ in $\mathbf{1}$, $\mathbf{a}$ and $\mathbf{b}$, respectively. Consequently, the reason why the $b$ parameter in $\mathbf{1}$ is significantly shorter than in a can only be explained by the difference between the $\mathrm{Zn}-\mathrm{N}-\mathrm{C}$ angles. Indeed, in 1, the Zn-N-C angles are 144(1) and $147(1)^{\circ}$ while they are 146.1(5) and 151.1(5) ${ }^{\circ}$ in a which leads to an increase of the $b$ parameter in a. If the $c$ parameter is bigger in $\mathbf{1}$ than in $\mathbf{a}$ and $\mathbf{b}$, is probably due to the additional crystallization water molecule which leads to stronger hydrogen bonds between the chains in both compounds a and $\mathbf{b}$.

In $2 \mathrm{Cu}^{2+}$ is fully coordinated by two ethylenediamines groups and one $\mathrm{H}_{2} \mathrm{O}$ molecule in a square-pyramidal coordination mode as depicted in Figure 4. 
Table 4. List of hydrogen bonds ( $\AA$ ) in compounds 1 and 2.

\begin{tabular}{llllll}
\hline$\left[\left\{\mathrm{Zn}\left(\mathrm{H}_{2} \mathrm{O}\right)(\mathrm{en})_{2}\right\}\left\{\mathrm{Zn}(\mathrm{en})_{2}\right\}-\mathrm{Mo}_{6} \mathrm{~B}_{\mathrm{r} 6} \mathrm{Se}_{2}(\mathrm{CN})_{6}\right] .2 \mathrm{H}_{2} \mathrm{O}(\mathbf{1})(\mathrm{D}-\mathrm{H}---\mathrm{A})(\AA)$ \\
$\mathrm{N} 2-\mathrm{H}---\mathrm{O} 2 \mathrm{cw}$ & $2.8352(4)$ & $\mathrm{N} 5-\mathrm{H}---\mathrm{N} 11$ & $3.09(2)$ & $\mathrm{N} 13-\mathrm{H}---\mathrm{L} 6$ & $3.42(1)$ \\
$\mathrm{N} 4-\mathrm{H}---\mathrm{O} 3 \mathrm{cw}$ & $2.7943(2)$ & $\mathrm{N} 7-\mathrm{H}---\mathrm{O} 3 \mathrm{cw}$ & $3.12(2)$ & $\mathrm{N} 14-\mathrm{H}---\mathrm{L} 4$ & $3.50(1)$ \\
$\mathrm{N} 4-\mathrm{H}---\mathrm{N} 12$ & $3.36(2)$ & $\mathrm{O} 3 \mathrm{cw}-\mathrm{H}---\mathrm{N} 12$ & $3.83(2)$ & $\mathrm{O} 2 \mathrm{cw}-\mathrm{H}---\mathrm{O} 3$ & $2.9091(4)$ \\
$\mathrm{N} 5-\mathrm{H}---\mathrm{O} 1$ & $3.0912(3)$ & $\mathrm{N} 9-\mathrm{H}---\mathrm{L} 3$ & $3.83(1)$ & & \\
\hline$\left[\left\{\mathrm{Cu}\left(\mathrm{H}_{2} \mathrm{O}\right)(\mathrm{en})_{2}\right\}_{2} \mathrm{Mo}_{6} \mathrm{Br}_{6} \mathrm{Se}_{2}(\mathrm{CN})_{6}\right] .2 \mathrm{H}_{2} \mathrm{O}(2)(\mathrm{D}-\mathrm{H}---\mathrm{A})(\AA)$ & & \\
\hline $\mathrm{N} 2-\mathrm{H}---\mathrm{N} 6$ & $3.0825(1)$ & $\mathrm{N} 6-\mathrm{H}---\mathrm{N} 3$ & $3.0489(1)$ & $\mathrm{O} 2-\mathrm{H}---\mathrm{N} 1$ & $2.8655(1)$ \\
$\mathrm{N} 3-\mathrm{H}---\mathrm{N} 5$ & $3.1207(1)$ & $\mathrm{N} 2-\mathrm{H}---\mathrm{O} 1$ & $3.2781(1)$ & $\mathrm{O} 2-\mathrm{H}---\mathrm{N} 5$ & $3.1164(1)$ \\
$\mathrm{N} 1-\mathrm{H}---\mathrm{N} 7$ & $3.0601(1)$ & & & \\
\hline
\end{tabular}

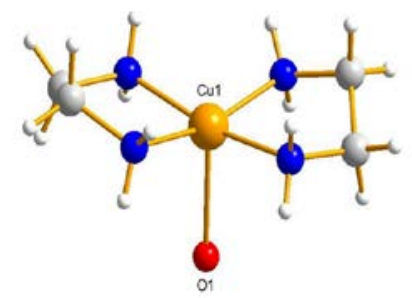

Figure 4. Representation of the $\mathrm{Cu} 1$ environment in $2 . \mathrm{Cu}^{2+}$ is coordinated by two ethylenediamine groups and one $\mathrm{H}_{2} \mathrm{O}$ molecule in a square-pyramidal coordination mode.

Square-pyramidal arrangement is common for $\mathrm{Cu}^{2+}$ and it is also found in other coordination polymers based on $\mathrm{Re}_{6}$ cyanide clusters [41-42]. In 2, the $\left[\mathrm{Mo}_{6} \mathrm{Br}_{6}^{\mathrm{i}} \mathrm{Se}_{2}^{\mathrm{i}}(\mathrm{CN})_{6}^{\mathrm{a}}\right]^{4-}$ cluster units are located on the vertices of the unit cell and in the middle of the faces defined by $b$ and $c$ parameters. The structure can be depicted as a AAA stacking type of hexagonal compact layers of $\left[\mathrm{Mo}_{6} \mathrm{Br}_{6}{ }_{6} \mathrm{Se}_{2}^{\mathrm{i}}(\mathrm{CN})_{6}^{\mathrm{a}}\right]^{4-}$ cluster units. Such a stacking generates prismatic sites in which the $\left[\mathrm{Cu}\left(\mathrm{H}_{2} \mathrm{O}\right)(\mathrm{en})_{2}\right]^{2+}$ complexes cations are located (Figure $5 \mathrm{a}$ ). It forms layers of cations intercalated between the cluster units layers (Figure 5b).
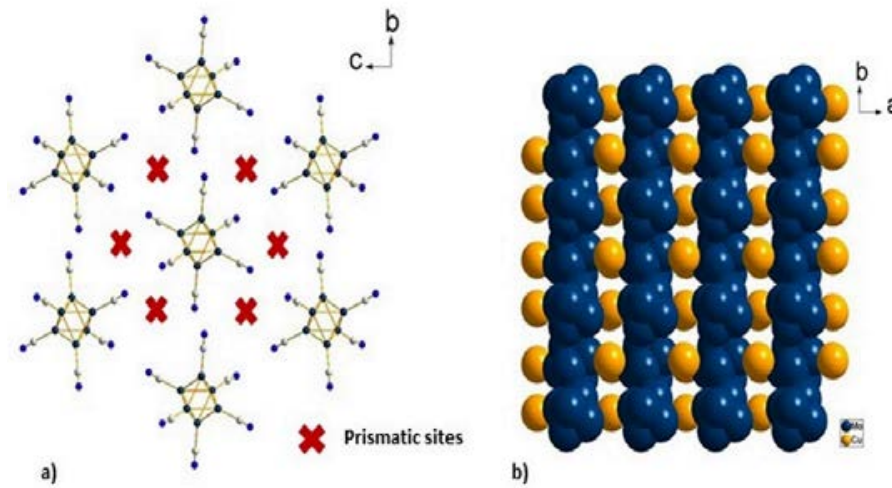

Figure 5. a) Projection in the $(b, c)$ plane of the pseudo hexagonal compact arrangement of the $\left[\mathrm{Mo}_{6} \mathrm{Br}_{6}{ }_{6}^{\mathrm{i}} \mathrm{Se}_{2}^{\mathrm{i}}(\mathrm{CN})_{6}^{\mathrm{a}}\right]^{4-}$ cluster units in $\mathbf{2}$. The red cross represent the prismatic sites which are generated by the AAA stacking type of units. Inner ligands, $\left[\mathrm{Cu}\left(\mathrm{H}_{2} \mathrm{O}\right)(\mathrm{en})_{2}\right]^{2+}$ complexes cations and crystallization water molecules have been omitted for clarity. b) Space filling model of the projection of the AAA stacking type of the $\left[\mathrm{Mo}_{6} \mathrm{Br}_{6}{ }_{6} \mathrm{Se}_{2}^{\mathrm{i}}(\mathrm{CN})_{6}^{\mathrm{a}}\right]^{4-}$ cluster units layers in the $(a, b)$ plane evidencing the alternating of $\left[\mathrm{Cu}\left(\mathrm{H}_{2} \mathrm{O}\right)(\mathrm{en})_{2}\right]^{2+}$ complexes cations layers and $\left[\mathrm{Mo}_{6} \mathrm{Br}_{6}{ }_{6} \mathrm{Se}_{2}^{\mathrm{i}}(\mathrm{CN})_{6}^{\mathrm{a}}\right]^{4-}$ cluster units layers. Only $\mathrm{Mo}_{6}$ octahedral clusters and $\mathrm{Cu}^{2+}$ cations are represented for the sake of clarity.

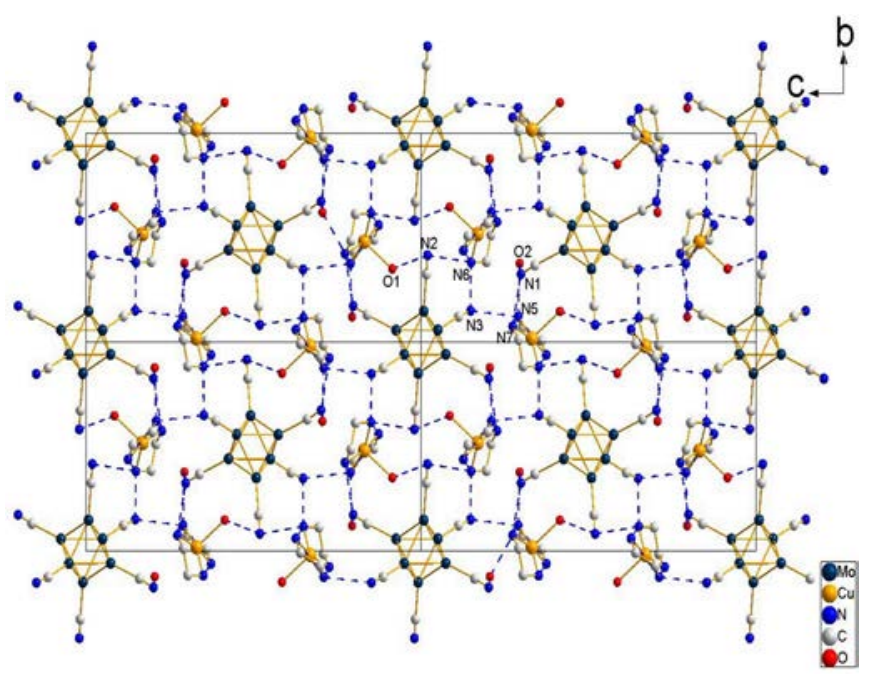

Figure 6. Projection in the (b, c) plane of a cluster units layer and the hydrogen bonds network between $\left[\mathrm{Mo}_{6} \mathrm{Br}_{6}{ }_{6} \mathrm{Se}_{2}{ }_{2}^{\mathrm{i}}(\mathrm{CN})_{6}^{\mathrm{a}}\right]^{4-}$ cluster units, $\left[\mathrm{Cu}\left(\mathrm{H}_{2} \mathrm{O}\right)(\mathrm{en})_{2}\right]^{2+}$ complexes cations and crystallization water molecules in $\mathbf{2}$. $\mathrm{H}$ atoms of the ethylenediamine groups and inner ligands have been omitted for clarity. 
In the infrared spectra, the single band at $2104 \mathrm{~cm}^{-1}$ indicates that all the $\mathrm{CN}$ ligands are free. Hence, the cations interact with $\left[\mathrm{Mo}_{6} \mathrm{Br}_{6}^{\mathrm{i}} \mathrm{Se}_{2}^{\mathrm{i}}{ }_{2}(\mathrm{CN})_{6}^{\mathrm{a}}\right]^{4-}$ cluster units via hydrogen bonds between cyanides ligands and nitrogen atoms of ethylenediamine groups (N2-N6 3.0825(1); N3-N5 3.1207(1); N6-N3 3.0489(1); N1-N7 3.0601(1) Å) and between cyanides ligands and the water molecule of the $\left[\mathrm{Cu}\left(\mathrm{H}_{2} \mathrm{O}\right)(\mathrm{en})_{2}\right]^{2+}$ complexes (N2-O1 3.2781(1) $\AA$ ). Crystallization water molecules also strongly participate to the structural cohesion via hydrogen bonds with cyanides ligands (O2cw-N1 2.8655(1) $\AA$ ) and with nitrogen atoms of ethylenediamine groups (O2cw-N5 3.1164(1) Å). These hydrogen bond distances are similar than those in $\left[\mathrm{Cu}(\mathrm{En})_{2}\left(\mathrm{H}_{2} \mathrm{O}\right)_{2}\right]\left[\left\{\mathrm{Cu}(\mathrm{En})_{2}\right\} \operatorname{Re}_{6} \mathrm{~S}_{8}(\mathrm{CN})_{4}(\mathrm{OH})_{2}\right] \cdot 5.5 \mathrm{H}_{2} \mathrm{O}$ and $\left[\mathrm{Cu}(\mathrm{En})_{2}\left(\mathrm{H}_{2} \mathrm{O}\right)_{2}\left[\left\{\mathrm{Cu}(\mathrm{En})_{2}\right\} \mathrm{Re}_{6} \mathrm{Se}_{8}(\mathrm{CN})_{4}(\mathrm{OH})_{2}\right] \cdot 5 \mathrm{H}_{2} \mathrm{O}[11 \mathrm{~b}]\right.$. This hydrogen bonds (Table 4) network between $\left[\mathrm{Mo}_{6} \mathrm{Br}_{6}{ }_{6} \mathrm{Se}_{2}^{\mathrm{i}}(\mathrm{CN})_{6}^{\mathrm{a}}\right]^{4-}$ cluster units, $\left[\mathrm{Cu}\left(\mathrm{H}_{2} \mathrm{O}\right)(\mathrm{en})_{2}\right]^{2+}$ complexes cations and crystallization water molecules (Figure 6) connects the cluster units layers together leading to a three-dimensional supramolecular framework as depicted in Figure 7.

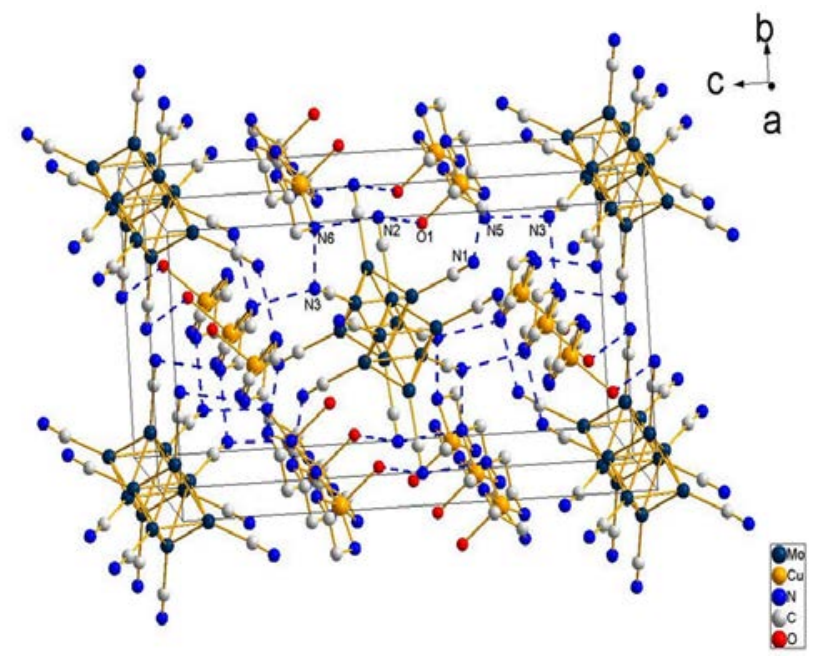

Figure 7. Representation of the hydrogen bonds allowing the connection between the cluster units layers and leading to a three-dimensional supramolecular framework. Inner ligands, hydrogen atoms and crystallization water molecules have been omitted for clarity.

The structure of $\mathbf{2}$ is strongly related to that of $\left[\mathrm{Ni}\left(\mathrm{NH}_{3}\right)_{4}(\mathrm{en})\right]_{2}\left[\mathrm{Re}_{6} \mathrm{Te}_{8}(\mathrm{CN})_{6}\right] \cdot 2 \mathrm{H}_{2} \mathrm{O} \quad[22-23]$ which crystallizes in the monoclinic space group $P 2_{1} / n$ with refined parameters $a=10.3607(7) \AA, b=10.6377(7) \AA, c=$ 21.2875(14) $\AA, \beta=97.170(1)^{\circ}$ and $V=2327.8(3) \AA^{3}$. In this compound, $\mathrm{Ni}^{2+}$ is fully coordinated by four ammonia molecules and one ethylenediamine group in an octahedral conformation type while in $\mathbf{2}, \mathrm{Cu}^{2+}$ is fully coordinated by two ethylenediamine groups and one water molecule in a square-pyramidal conformation type. Square-pyramidal coordination type for $\mathrm{Cu}^{2+}$ is also found in $\left[\left\{\mathrm{Cu}(\mathrm{en})_{2}\right\}_{2} \mathrm{Re}_{6} \mathrm{Te}_{8}(\mathrm{CN})_{6}\right] .5 \mathrm{H}_{2} \mathrm{O}$ [42]. However, in the latter, a $\mathrm{CN}$ group of the cluster completes the coordination sphere of the $\mathrm{Cu}$ instead of a water molecule in 2 , leading to a neutral complex $\left[\left\{\mathrm{Cu}(\mathrm{en})_{2}\right\}_{2} \mathrm{Re}_{6} \mathrm{Te}_{8}(\mathrm{CN})_{6}\right]$. In both compounds, the $\mathrm{Cu}-\mathrm{N}(\mathrm{en})$ distances are close and range from
2.003(7) to 2.039(7) $\AA$, in 2, and from 2.011(6) to 2.037(4) $\AA$, in $\left[\left\{\mathrm{Cu}(\mathrm{en})_{2}\right\}_{2} \mathrm{Re}_{6} \mathrm{Te}_{8}(\mathrm{CN})_{6}\right] \cdot 5 \mathrm{H}_{2} \mathrm{O}$.

\section{CONCLUSION}

Two new compounds $\left[\left\{\mathrm{Zn}\left(\mathrm{H}_{2} \mathrm{O}\right)(\mathrm{en})_{2}\right\}\left\{\mathrm{Zn}(\mathrm{en})_{2}\right\}\right.$ $\left.\mathrm{Mo}_{6} \mathrm{~B}_{\mathrm{r6}} \mathrm{Se}_{2}(\mathrm{CN})_{6}\right] \cdot 2 \mathrm{H}_{2} \mathrm{O}$ (1) and $\left[\left\{\mathrm{Cu}\left(\mathrm{H}_{2} \mathrm{O}\right)(\mathrm{en})_{2}\right\}_{2} \mathrm{Mo}_{6} \mathrm{Br}_{6} \mathrm{Se}_{2}(\mathrm{CN})_{6}\right] \cdot 2 \mathrm{H}_{2} \mathrm{O} \quad$ (2) (en = ethylenediamine) have been obtained by the reaction of $\mathrm{Cs}_{2} \mathrm{~K}_{2}\left[\mathrm{Mo}_{6} \mathrm{Br}_{6} \mathrm{Se}_{2}(\mathrm{CN})_{6}\right]$ with $\mathrm{Zn}\left[\mathrm{OOCCH}_{3}\right]_{2} \cdot 2 \mathrm{H}_{2} \mathrm{O}$ and ethylenediamine, and with $\mathrm{CuCl}_{2} \cdot \mathrm{xH}_{2} \mathrm{O}$ and ethylenediamine for $\mathbf{1}$ and 2, respectively. For the synthesis of 2, ammonia has been used as solvant to organize a competition between ammonia and ethylenediamine as chelating ligands in order to slow down the reaction and induce crystallization. Both structures have been structurally characterized by singlecrystal X-ray diffraction. $\mathbf{1}$ is a bimetallic one dimensional coordination compound while $\mathbf{2}$ is a bimetallic $\mathrm{H}$ bonded three dimensional compound. Structural cohesion of both compounds is mainly ensured by hydrogen bonds. The use of ethylenediamine as ligand permits hydrogen bonds especially between cyanides ligands and nitrogen atoms of ethylenediamine groups in both compounds which participate to the stability of the structures. Further work will consist in obtaining microcrystalline powder of both compounds to make characterizations as TGA and thermal dependent X-ray powder diffraction in order to study the thermal stability of the compounds and possible structural modifications.

\section{ACKNOWLEDGEMENTS}

The authors thank the International Associate Laboratory CLUSPOM (2014-2017). Gilles Daigre thanks "Ecole des Docteurs de l'Université Bretagne Loire" and the "Conseil Regional de Bretagne" for the scholarship mobility at the Nikolaev Institute of Inorganic Chemistry of Novosibirsk. The authors also thank the "Centre de diffractométrie X" of the Institute of Chemical Science of Rennes for singlecrystal X-ray diffraction data collection.

\section{SUPPLEMENTARY MATERIAL}

Further details of the crystal structures investigations may be obtained from the Fachinformationszentrum Karlsruhe, 76344 Eggenstein- Leopoldshafen, Germany (Fax: +497247-808-666; E-Mail: $\quad$ crysdata@fiz-karlsruhe.de, http://www.fiz karlsruhe.de/request for deposited data.html) on quoting the depository numbers CSD- 432616 and CSD432617.

\section{REFERENCES}

[1] Buser, H.J.; Schwarzenbach, D.; Petter, W.; Ludi, A. The crystal Structure of Prussian Blue $\mathrm{Fe}_{4}\left[\mathrm{Fe}(\mathrm{CN})_{6}\right]_{3} \cdot \mathrm{xH}_{2} \mathrm{O}$. Inorg. Chem.. 1977, Vol. 16, No. 11, 2704-2710.

[2] Jiang, M.; Zhao, Z. A novel stable electrochromic thin film: a Prussian Blue analogue based on palladium hexacyanoferrate. J. Electroanal. Chem. 1990, 292, 281-287.

[3] Ferlay, S.; Mallah, T.; Ouahès, R.; Verdaguer, M.; Veillet, P. A roomtemperature organometallic magnet based on Prussian blue. Nature. 1995, 378, 701-703. 
[4] Naumov, N.G.; Virovets, A.V.; Sokolov, M.N.; Artemkina, S.B.; Fedorov, V.E. A Novel Framework Type Inorganic Clusters with Cyanide Ligands: Crystal Structure of $\mathrm{Cs}_{2} \mathrm{Mn}_{3}\left[\mathrm{Re}_{6} \mathrm{Se}_{8}(\mathrm{CN})_{6}\right]_{2} \cdot 15 \mathrm{H}_{2} \mathrm{O}$ and $\left(\mathrm{H}_{3} \mathrm{O}\right)_{2} \mathrm{Co}_{3}\left[\mathrm{Re}_{6} \mathrm{Se}_{8}(\mathrm{CN})_{6}\right]_{2} .14 .5 \mathrm{H}_{2} \mathrm{O}$. Angew. Chem. Int. Ed. 1998, 37.

[5] Beauvais, L.G.; Shores, M.P.; Long, J.R. Cyano-Bridged $\mathrm{Re}_{6} \mathrm{Q}_{8}(\mathrm{Q}=\mathrm{S}$, Se) Cluster-Metal Framework Solids: A New Class of Porous Materials. Chem. Mater. 1998, 10, 3783-3786.

[6] Shores, M.P.; Beauvais, L.G.; Long, J.R. $\left[\mathrm{Cd}_{2}\left(\mathrm{H}_{2} \mathrm{O}\right)_{4}\right]\left[\mathrm{Re}_{6} \mathrm{~S}_{8}(\mathrm{CN})_{6}\right] \cdot 14 \mathrm{H}_{2} \mathrm{O}$ : A Cyano-Bridged Cluster-Cluster Framework Solid with Accessible Cubelike Cavities. Inorg. Chem. 1999, 38, 1648-1649.

[7] Beauvais, L.G.; Shores, M.P.; Long, J.R. Cyano-Bridged $\mathrm{Re}_{6} \mathrm{Q}_{8}(\mathrm{Q}=\mathrm{S}$, Se) Cluster-Cobalt(II) Framework Materials: Versatile Solid Chemical Sensors. J. Am. Chem. Soc. 2000, 122, 2763-2772.

[8] Benett, M.V.; Shores, M.P.; Beauvais, L.G.; Long, J.R. Expansion of the Porous Solid $\mathrm{Na}_{2} \mathrm{Zn}_{3}\left[\mathrm{Fe}(\mathrm{CN})_{6}\right]_{2} \cdot 9 \mathrm{H}_{2} \mathrm{O}$ : Enhanced Ion-Exchange Capacity in $\mathrm{Na}_{2} \mathrm{Zn}_{3}\left[\mathrm{Re}_{6} \mathrm{Se}_{8}(\mathrm{CN})_{6}\right]_{2} \cdot 24 \mathrm{H}_{2} \mathrm{O}$. J. Am. Chem. Soc. 2000, 122, 6664-6668.

[9] Benett, M.V.; Beauvais, L.G.; Shores, M.P.; Long, J.R. Expanded Prussian Blue Analogues Incorporating $\left[\mathrm{Re}_{6} \mathrm{Se}_{8}(\mathrm{CN})_{6}\right]^{3-1 / 4}$ Clusters: Adjusting Porosity via Charge Balance. J. Am. Chem. Soc. 2001, 123, 80228032.

[10] Fedorov, V.E.; Naumov, N.G.; Mironov, Y.V.; Virovets, A.V.; Artemkina, S.B., Brylev, K.A.; Yarovoi, S.S.; Efremoa, O.A.; Pack, U-H. Inorganic coordination polymers based on chalcocyanide cluster complexes. J. Struct. Chem.. 2002, 43, 669-684.

[11] Naumov, N.G.; Virovets, A.V.; Fedorov, V.E. Unusually high porosity in polymeric cluster cyanides: the synthesis and crystal structure of $\left(\mathrm{H}_{3} \mathrm{O}\right)_{2} \mathrm{Zn}_{3}\left[\mathrm{Re}_{6} \mathrm{Se}_{8}(\mathrm{CN})_{6}\right]_{2} .20 \mathrm{H}_{2} \mathrm{O}$. Inorg. Chem. Commun. 3. 2000, 71-72.

[12] Mironov, Y.V.; Oeckler, O.; Simon, A.; Fedorov, V.E. New Types of Complexes Based on $\mathrm{Re}_{4}$ Chalcocyanide Clusters - Syntheses and Crystal Structures of $\left[\mathrm{Ni}\left(\mathrm{NH}_{3}\right)_{5}\right]_{2}\left[\mathrm{Re}_{4} \mathrm{Te}_{4}(\mathrm{CN})_{12}\right] \cdot 3.4 \mathrm{H}_{2} \mathrm{O}$ and $\left[\mathrm{Cd}\left(\mathrm{NH}_{3}\right)_{5}\right]\left[\mathrm{Cd}\left(\mathrm{NH}_{3}\right)_{3}\right]\left[\mathrm{Re}_{4} \mathrm{Te}_{4}(\mathrm{CN})_{12}\right] \cdot 4 \mathrm{H}_{2} \mathrm{O}$. Eur. J. Inorg. Chem. 2001, 2751-2753.

[13] Mironov, Y.V.; Efremova, O.A.; Naumov, D.Y.; Sheldrick, W.S.; Fedorov, V.E. Synthesis and Structures of Compounds Based on Chalcocyanide Tetranuclear Rhenium Clusters: Bonding Cluster Complexes by diene-Bridged $\mathrm{Cu}^{\mathrm{II}}$ Units. Eur. J. Inorg. Chem. 2003, 2591-2595.

[14] Brylev, K.A.; Naumov, N.G.; Peris, G.; Llusar, R.; Fedorov V. E. Novel inorganic ionic compounds based on $\mathrm{Re}_{6}$ chalcocyanide cluster complexes: synthesis and crystal structures of $\left[\mathrm{CuNH}_{3} \text { (trien) }\right]_{2}\left[\mathrm{Re}_{6} \mathrm{~S}_{8}(\mathrm{CN})_{6}\right] \cdot 7 \mathrm{H}_{2} \mathrm{O}, \quad\left[\mathrm{CuNH}_{3}(\text { trien })\right]_{2}\left[\mathrm{Re}_{6} \mathrm{Se}_{8}(\mathrm{CN})_{6}\right] \quad$ and $\left[\mathrm{CuNH}_{3} \text { (trien) }\right]_{2}\left[\mathrm{Re}_{6} \mathrm{Te}_{8}(\mathrm{CN})_{6}\right] \cdot \mathrm{H}_{2} \mathrm{O}$. Polyhedron. 2003, 22, 3383-3387.

[15] Kim, Y.; Park, S.-M.; Nam, W.; Kim, S.-J. New Compounds from Tellurocyanide Rhenium Cluster Anions and 3d-Transition Metal Cations Coordinated with Ethylenediamine. Chem. Commun. 2001, 1470-1471.

[16] Y. Kim, S.-M. Park, S.-J. Kim Three-dimensional framework containing $\mathrm{Mn}(\mathrm{salen})^{+}$and $\mathrm{Re}_{6} \mathrm{Se}_{8}(\mathrm{CN})_{6}{ }^{4-}$ cluster. Inorg. Chem. Commun. 2002, 5, 592-595.

[17] Naumov, N.G.; Virovets, A.V.; Mironov, Y.I.; Artemkina, S.B.; Fedorov, V.E. Synthesis and crystal structure of new layered cluster cyanides $\mathrm{Cs}_{2} \mathrm{M}\left[\mathrm{Re}_{6} \mathrm{~S}_{8}(\mathrm{CN})_{6}\right] \cdot 2 \mathrm{H}_{2} \mathrm{O}\left(\mathrm{M}=\mathrm{Mn}^{2+}, \mathrm{Fe}^{2+}, \mathrm{Co}^{2+}, \mathrm{Cd}^{2+}\right)$ : size control over framework dimension. Ukr. Khim. Zh. 1999, 65, 21-27.

[18] Jin, S.; Disalvo, F.J. 3-D Coordination Network Structures Constructed from $\left[\mathrm{W}_{6} \mathrm{~S}_{8}(\mathrm{CN})_{6}\right]^{6-}$ Anions. Chem. Mater. 2002, 14, 3448-3457.

[19] Artemkina, S.B.; Naumov, N.G.; Virovets, A.V.; Oeckler, O.; Simon, A.; Erenburg, S.B.; Bausk, N.V.; Fedorov, V.E. Two Molecular-Type Complexes of the Octahedral Rhenium(III) Cyanocluster Anion $\left[\mathrm{Re}_{6} \mathrm{Se}_{8}(\mathrm{CN})_{6}\right]^{4+}$ with $\mathrm{M}^{2+}\left(\mathrm{Mn}^{2+}, \mathrm{Ni}^{2+}\right)$. Eur. J. Inorg. Chem. 2002, 11981202.

[20] Brylev, K. A.; Naumov, N. G.; Peris, G.; Llusar, R.; Fedorov, V.E. Novel inorganic ionic compounds based on $\mathrm{Re}_{6}$ chalcocyanide cluster complexes: synthesis and crystal structures of $\left[\mathrm{CuNH}_{3} \text { (trien) }\right]_{2}\left[\mathrm{Re}_{6} \mathrm{~S}_{8}(\mathrm{CN})_{6}\right] .7 \mathrm{H}_{2} \mathrm{O}, \quad\left[\mathrm{CuNH}_{3}(\text { trien })\right]_{2}\left[\mathrm{Re}_{6} \mathrm{Se}_{8}(\mathrm{CN})_{6}\right] \quad$ and $\left[\mathrm{CuNH}_{3} \text { (trien) }\right]_{2}\left[\mathrm{Re}_{6} \mathrm{Te}_{8}(\mathrm{CN})_{6}\right] . \mathrm{H}_{2} \mathrm{O}$. Polyhedron, 2003, 22, 3383-3387. [21] Naumov, N.G.; Virovets, A.V.; Artemkina, S.B.; Naumov, D.Y.; Howard, J.A.K.; Fedorov, V.E. A family of three-dimensional porous coordination polymers with general formula $(\text { Kat })_{2}\left[\left\{M\left(\mathrm{H}_{2} \mathrm{O}\right)_{n}\right\}_{3}\left\{\mathrm{Re}_{6} Q_{8}(\mathrm{CN})_{6}\right\}_{2}\right] \times \times \mathrm{H}_{2} \mathrm{O}(\mathrm{Q}=\mathrm{S}$, Se; $n=1.5$, 2). J. Solid State Chem. 2004, 177, 1896-1904.

[22] Brylev, K.A.; Mironov, Y.I.; Naumov, N.G.; Fedorov, V.E.; Ibers, J.A. New Compounds from Tellurocyanide Rhenium Cluster Anions and 3dTransition Metal Cations Coordinated with Ethylenediamine. Inorg. Chem. 2004, 43, 4833-4838.

[23] Ermolaev, A.V.; Smplentsev, A.I.; Mironov, Yu.V.; Polymers Based on Anionic Octahedral Cluster Chalcocyanohydroxorhenium Complexes and Cations $\left[\mathrm{Cu}(\mathrm{En})_{2}\right]^{2+}$. Russ. J. Coord. Chem.. 2014, 558-564.

[24] Simsek, M.K.; Bublitz, D.; Preetz, W. Darstellung, Kristallstrukturen, Schwingungsspektren und Normalkoordinatenanalyse von $\left[\left(\mathrm{Mo}_{6} \mathrm{Br}_{8}^{\mathrm{i}}\right) \mathrm{Y}_{6}^{\mathrm{a}}\right]^{2-}$; $\mathrm{Y}^{\mathrm{a}}=$ CN, NCS. Z. Anorg. Allg. Chem. 1997, 623, 1885-1891.

[25] Yan, B.; Zhou, H.; Lachgar, A. Octahedral Niobium Chloride Clusters as Building Blocks of Templated Prussian Blue Framework Analogues. Inorg. Chem. 2003, 42, 8818-8822.

[26] Naumov, N.G.; Cordier, S.; Perrin, C. An extended open framework based on disordered $\left[\mathrm{Nb}_{6} \mathrm{Cl}_{9} \mathrm{O}_{3}(\mathrm{CN})_{6}\right]^{5-}$ cluster units: Synthesis and crystal structure of $\mathrm{Cs}_{3} \mathrm{Mn}\left[\mathrm{Nb}_{6} \mathrm{Cl}_{9} \mathrm{O}_{3}(\mathrm{CN})_{6}\right] \cdot 0.6 \mathrm{H}_{2} \mathrm{O}$. Solid State Sci. 2005, 7, 15171521.

[27] Zhang, J.; Lachgar, A. Superexpanded Prussian-Blue Analogue with $\left[\mathrm{Fe}(\mathrm{CN})_{6}\right]^{4-},\left[\mathrm{Nb}_{6} \mathrm{Cl}_{12}(\mathrm{CN})_{6}\right]^{4-}$, and $[\mathrm{Mn}(\text { salen })]^{+}$as Building Units. J. Am. Chem. Soc. 2007, 129, 250-251.

[28] Amela-Cortes, M.; Cordier, S.; Naumov, N.G.; Meriadec, C.; Artzner, F.; Molard, Y. Hexacyano octahedral metallic clusters as versatile building blocks in the design of extended polymeric frameworks and clustomesognes. J. Mater. Chem. C. 2014, 2, 9813-9823.

[29] Cordier, S.; Naumov, N.G.; Salloum, D.; Paul, F.; Perrin, C. Synthesis and Characterization of $\mathrm{Mo}_{6}$ Chalcobromides and Cyano-Substituted Compounds Built from a Novel $\left[\left(\mathrm{Mo}_{6} \mathrm{Br}_{6}^{\mathrm{i}} \mathrm{Y}_{2}^{\mathrm{i}}\right) \mathrm{L}_{6}^{\mathrm{a}}\right]^{n-}$ Discrete Cluster Unit $\left(\mathrm{Y}^{\mathrm{i}}\right.$ $=\mathrm{S}$ or Se and $\mathrm{L}^{\mathrm{a}}=\mathrm{Br}$ or $\mathrm{CN}$ ). Inorg. Chem. 2004, 43, 219-226.

[30] Yunlu, K.; Hock, N.; Fischer, D. Polymeric Tris[trimethyltin(IV)]hexacyanocobaltate(III), a Compound Non-Analogous to "Super Prussian Blue," and Its Tris[tricyclopentadienyluranium(IV)] Homologue. Angew. Chem., Int. Ed. 1985, 24, 879-881.

[31] Siebel, E.; Fischer, R.D.; Davies, N.A.; Apperley, D.C.; Harris, R.K. The organometallic double metal cyanide $\left[\left(\mathrm{Me}_{2} \mathrm{Sn}\right)_{3}\left\{\mathrm{Co}(\mathrm{CN})_{6}\right\}_{2} \cdot 6 \mathrm{H}_{2} \mathrm{O}\right]$. A three-dimensional framework of infinite, stapled ribbons. J. Organomet. Chem. 2000, 604, 34-42.

[32] Schäfer, H.; Von schnering, H.G.; Tillack, J.; Kuhnen, F.; Worhle, H.; Baumann, H. Neue Untersuchungen über die Chloride des Molybdäns. $Z$. Anorg. Allg. Chem., 1967, 353, 281.

[33] APEX3 program suite V2016.1-0, Bruker AXS Inc., Wisconsin, USA.

[34] APEX2 program suite V2014.11-0, Bruker AXS Inc., Wisconsin, USA.

[35] Sheldrick, G.M. SAINT Version 8.37A, 2013, Bruker AXS Inc., Wisconsin, USA.

[36] Sheldrick, G.M. SADABS version 2014/5, SADABS Bruker AXS Inc., Madison, Wisconsin, USA.

[37] Sheldrick, G.M. SHELXT - Integrated space-group and crystalstructure determination. Acta Cryst. 2015, A71, 3-8.

[38] Sheldrick, G.M. Crystal structure refinement with SHELXL. Acta Cryst. 2015, C71, 3-8.

[39] Farrugia, L.J. WinGX and ORTEP for Windows: an uptade. J. Appl. Cryst. 2012, 45, 849.

[40] Brylev, K.A.; Pilet, G.; Naumov, N.G.; Perrin, A.; Fedorov, V.E. Structural Diversity of Low-Dimensional Compounds in $\left[\mathrm{M}(\mathrm{en})_{2}\right]^{2+} /\left[\mathrm{Re}_{6} \mathrm{Q}_{8}(\mathrm{CN})_{6}\right]^{4+}$ Systems $(\mathrm{M}=\mathrm{Mn}, \mathrm{Ni}, \mathrm{Cu})$. Eur. J. Inorg. Chem. 2005, 461-466.

[41] Mironov, Y.I.; Naumov, N.G.; Brylev, K.A.; Efremova, O.A.; Fedorov, V.E.; Hegetschweiler, K. Rhenium-Chalcogenide-Cyano Clusters, $\mathrm{Cu}^{2+}$ Ions, and 1,2,3,4-Tetraaminobutane as Molecular Building Blocks for Chiral Coordination Polymers. Angew. Chem. Int. Ed. 2004, 43, 1297-1300.

[42] Mironov, Y.V.; Fedorov, V.E.; Ijjaali, I.; Ibers, J.A. $\left[\left\{\mathrm{Cu}(\mathrm{en})_{2}\right\}_{2} \mathrm{Re}_{4} \mathrm{Te}_{4}(\mathrm{CN})_{12}\right] \cdot 5 \mathrm{H}_{2} \mathrm{O}$ and $\quad\left[\left\{\mathrm{Cu}(\mathrm{en})_{2}\right\}_{2} \mathrm{Re}_{6} \mathrm{Te}_{8}(\mathrm{CN})_{6}\right] \cdot 5 \mathrm{H}_{2} \mathrm{O}$ : Bonding of a Transition-Metal Complex to a Rhenium Chalcocyanide Cluster. Inorg. Chem. 2001, 40, 6320-6323. 